\title{
MixONat, a software for the dereplication of mixtures based on ${ }^{13} \mathrm{C}$ NMR spectroscopy
}

\author{
Antoine Bruguière ${ }^{\dagger}$, Séverine Derbrét ${ }^{*}$, Joël Dietsch ${ }^{+, \neq}$, Jules Leguy, Valentine Rahier ${ }^{\S}$, Quentin \\ Pottier $^{\dagger}$, Dimitri Bréard ${ }^{\dagger}$, Sorphon Suor-Cherer ${ }^{\dagger}$, Guillaume Viault ${ }^{\dagger}$, Anne-Marie Le Ray ${ }^{\dagger}$, Frédéric \\ Saubion ${ }^{\S}$, Pascal Richomme ${ }^{+*}$ \\ + SONAS, EA921, UNIV Angers, SFR QUASAV, Faculty of Health Sciences, Dpt Pharmacy, 16 Bd Daviers, 49045 Angers cedex 01, \\ France \\ ${ }^{\ddagger}$ JEOL Europe SAS, 1 Allée de Giverny, 78290 Croissy-sur-Seine, France \\ ${ }^{\S}$ LERIA, EA2645, UNIV Angers, SFR MathSTIC, Faculty of Sciences, 2 boulevard Lavoisier, 49045 Angers cedex 01, France \\ * Corresponding authors: severine.derbre@univ-angers.fr; pascal.richomme@univ-angers.fr
}

\section{Table of contents}

Figure S1. Third tab of the MixONat software corresponding to the C-TypeGen subprogram which sorts chemical shifts according to carbon types. C-TypeGen requires the original DB (.sdf) and the same one without stereochemistry informations. A new .sdf is created with, for each compound of the DB, predicted $\delta c$ values sorted out as methyl, methylene, methine or quaternary carbons.

Figure S2. Home tab of the MixONat software. The input files must include at least one database (.sdf) processed by the CtypeGen routine, and ${ }^{13} \mathrm{C}-\mathrm{NMR}$ data, imported as a table (.csv) of $\delta_{\mathrm{C}}$ values and intensities. DEPT-135 and 90 data can also be provided as tables (.csv) of $\delta_{\mathrm{C}}$ values and intensities.

Figure S3. Second tab of the MixONat software. It displays all the different parameters that can be adjusted including the tolerance $(\varepsilon)$ (Incrementation ON or OFF), the DEPT alignment parameter; the equivalent carbons option, the molecular weight filter and the minimal score required. The number of results and the directory path for saving results can also be selected in this tab.

Figure S4. Display of results for the ${ }^{13} \mathrm{C}-\mathrm{NMR}$ dereplication (+ DEPT 90 and 135) of the poppy extract. Compounds of the DB are ranked by decreasing score and increasing error, with their structure, name, molecular weight, score and error. On the structure, matched carbons are highlighted in red.

Figure S5. Graphical representation available for each compound (here papaverine from the poppy extract) identified in the DB. A reconstituted ${ }^{13} \mathrm{C}-\mathrm{NMR}$ spectrum shows intensities (with different color types) for matched carbons...................... S7

Figure S6. Part of displayed results for the ${ }^{13} \mathrm{C}$-NMR dereplication of the poppy extract (+ DEPT 90 and 135) saved as text

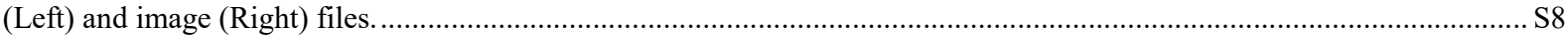

Figure S7. HPLC-UV $(\lambda 230 \mathrm{~nm})$ chromatogram of the Papaver somniferum alkaloids extract ....................................... S9

Figure S8. ${ }^{13} \mathrm{C}-\mathrm{NMR}$ spectrum (17408 scans) of the poppy alkaloids extract (10 mg) recorded in $\mathrm{CDCl}_{3}$ at $100 \mathrm{MHz} \ldots \ldots . . . \mathrm{S} 10$

Figure S9. Part of displayed results for the ${ }^{13} \mathrm{C}$-NMR dereplication of the poppy extract $\left({ }^{13} \mathrm{C}-\mathrm{NMR}\right.$ only)........................ S11

Figure S10. Part of displayed results for the ${ }^{13} \mathrm{C}-\mathrm{NMR}$ dereplication (+ DEPT- 90 and 135) of the poppy extract. ............. S11

Figure S11. HPLC-ELSD chromatogram of the Rosmarinus officinalis $\mathrm{MeOH}$ extract.

Figure S12. Part of displayed results for the ${ }^{13} \mathrm{C}-\mathrm{NMR}$ dereplication (+ DEPT 90 and 135) of the rosemary MeOH extract. Equivalent carbons were allowed.

Figure S13. ${ }^{13} \mathrm{C}-\mathrm{NMR}$ spectrum (2048 scans) of the rosemary leaf $\mathrm{MeOH}$ extract (50 mg) recorded in methanol-d 4 at $100 \mathrm{MHz}$

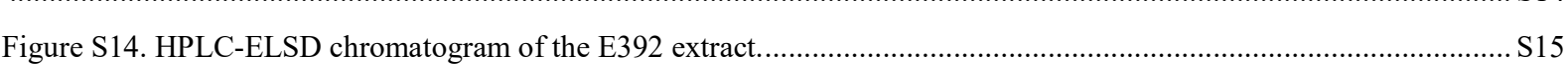

Figure S15. Structures of terpenes from E392 extract

Figure S16. Part of displayed results for the ${ }^{13} \mathrm{C}-\mathrm{NMR}$ dereplication (+ DEPT 90 and 135) of the rosemary E392 extract. Equivalent carbons were allowed. A molecular weight filter ( $>250 \mathrm{Da})$ was applied.

Figure S17. Part of displayed results for the ${ }^{13} \mathrm{C}-\mathrm{NMR}$ dereplication (+ DEPT 90 and 135) of the rosemary E392 extract. Equivalent carbons were allowed. Only NPs with MW 330, 332, 454 or 456 Da were allowed. 
Figure S18. ${ }^{13} \mathrm{C}-\mathrm{NMR}$ spectrum (1024 scans) of the rosemary E392 extract (50 mg) recorded in DMSO-d6 at $100 \mathrm{MHz} \ldots . . \mathrm{S} 18$

Figure S19. HPLC-ELSD chromatogram of the Rosmarinus officinalis DCM extract.

Figure S20. Part of displayed results for the ${ }^{13} \mathrm{C}-\mathrm{NMR}$ dereplication (+ DEPT 90 and 135) of the rosemary DCM extract. Equivalent carbons were allowed Only NPs with MW 330, 332, 454 or 456 Da were allowed..... S20

Figure S21. ${ }^{13} \mathrm{C}$-NMR spectrum (10000 scans) of the rosemary leaf DCM extract (50 mg) recorded in DMSO-d6 at $100 \mathrm{MHz}$ .. S21

Figure S22. (A) HPLC-UV ( $\lambda 254 \mathrm{~nm}$ ) chromatogram of the Garcina mangostana (fruit peel) cyclohexanic extract (B) \% (area) of each xanthone peak. (C) Structures of G. mangostana xanthones

Figure S23. Part of displayed results for the ${ }^{13} \mathrm{C}-\mathrm{NMR}$ dereplication (+ DEPT 90 and 135) of the mangosteen (fruit peel) cyclohexanic extract. Equivalent carbons were allowed.

Figure S24. ${ }^{13} \mathrm{C}$-NMR spectrum (10000 scans) of the mangosteen (fruit peel) cyclohexanic extract (30 mg) recorded in $\mathrm{CDCl}_{3}$ at $100 \mathrm{MHz}$.

Figure S25. DEPT-90 spectrum (5000 scans) of the mangosteen fruit peel cyclohexanic extract ( $30 \mathrm{mg}$ ) recorded in $\mathrm{CDCl}_{3}$ at $100 \mathrm{MHz}$.

Figure S26. ${ }^{13} \mathrm{C}-\mathrm{NMR}$ spectrum (10000 scans) of the mangosteen (fruit peel) cyclohexanic extract (30 mg) recorded in $\mathrm{CDCl}_{3}$ at $175 \mathrm{MHz}$.

Figure S27. Part of displayed results for the ${ }^{13} \mathrm{C}-\mathrm{NMR}$ dereplication (+ DEPT 90 and 135) (175 MHz) of the mangosteen (fruit peel) fraction based on Garcinia DB. Equivalent carbons were allowed.

Figure S28. HPLC-UV ( $\lambda 254 \mathrm{~nm}$ ) chromatogram of the fraction from the Garcina mangostana (fruit peel) cyclohexanic extract containing minor xanthones. ... S28

Figure S29. Part of displayed results for the ${ }^{13} \mathrm{C}-\mathrm{NMR}$ dereplication (+ DEPT 90 and 135) of the mangosteen (fruit peel) fraction based on Garcinia DB. Equivalent carbons were allowed. S28

Figure S30. ${ }^{13} \mathrm{C}-\mathrm{NMR}$ spectrum (12000 scans) of the fraction from mangosteen (fruit peel) cyclohexanic extract (30 mg) containing minor xanthones recorded in $\mathrm{CDCl}_{3}$ at $100 \mathrm{MHz}$...... S29

Figure S31. Part of displayed results for the ${ }^{13} \mathrm{C}-\mathrm{NMR}$ dereplication (+ DEPT 90 and 135) of the mangosteen (fruit peel) fraction based on CH-NMR-NP DB. Equivalent carbons were allowed. S30

Table S1. (-)-noscapine $\delta_{\mathrm{C}}$ in the poppy extract and predicted (ACD/Labs NMR Predictor) values. $\Delta \delta_{\mathrm{C}}>1.3 \mathrm{ppm}$ are highlighted in yellow.

Table S2. ${ }^{13} \mathrm{C}$ NMR spectrum of papaverine recorded in $\mathrm{CDCl}_{3}$ at $100 \mathrm{MHz}$.

Table S3. Retention time $\left(\mathrm{R}_{\mathrm{t}}\right)$ and ESI-MS² data for the major compounds identified in the rosemary leaf MeOH extract... S33

Table S4. Retention time $\left(\mathrm{R}_{\mathrm{t}}\right)$ and ESI-MS ${ }^{2}$ data for the major compounds identified in the rosemary leaf DCM and E392 extracts

Table S5. $\delta$ c matching with triterpenes and carnosic acid in the E392 extract along with their respective predicted values (ACD/Labs NMR Predictor). ${ }^{1} \Delta \delta_{\mathrm{C}}>1.3 \mathrm{ppm}$ are highlighted in yellow. Hidden and missing chemical shifts are highlighted in green and blue respectively. ... S34

Table S6. ${ }^{13} \mathrm{C}$ NMR spectrum of carnosol recorded in DMSO-d6 at $100 \mathrm{MHz}$ S36

Table S7. $\delta \mathrm{c}(100 \mathrm{MHz})$ matching with xanthones in the mangosteen cyclohexanic extract along with their respective predicted values (ACD/Labs NMR Predictor). $\Delta \delta_{\mathrm{C}}>1.3 \mathrm{ppm}$ are highlighted in yellow. Hidden and missing chemical shifts are highlighted in green and blue respectively. .. S37

Detailed MixONat software S43 


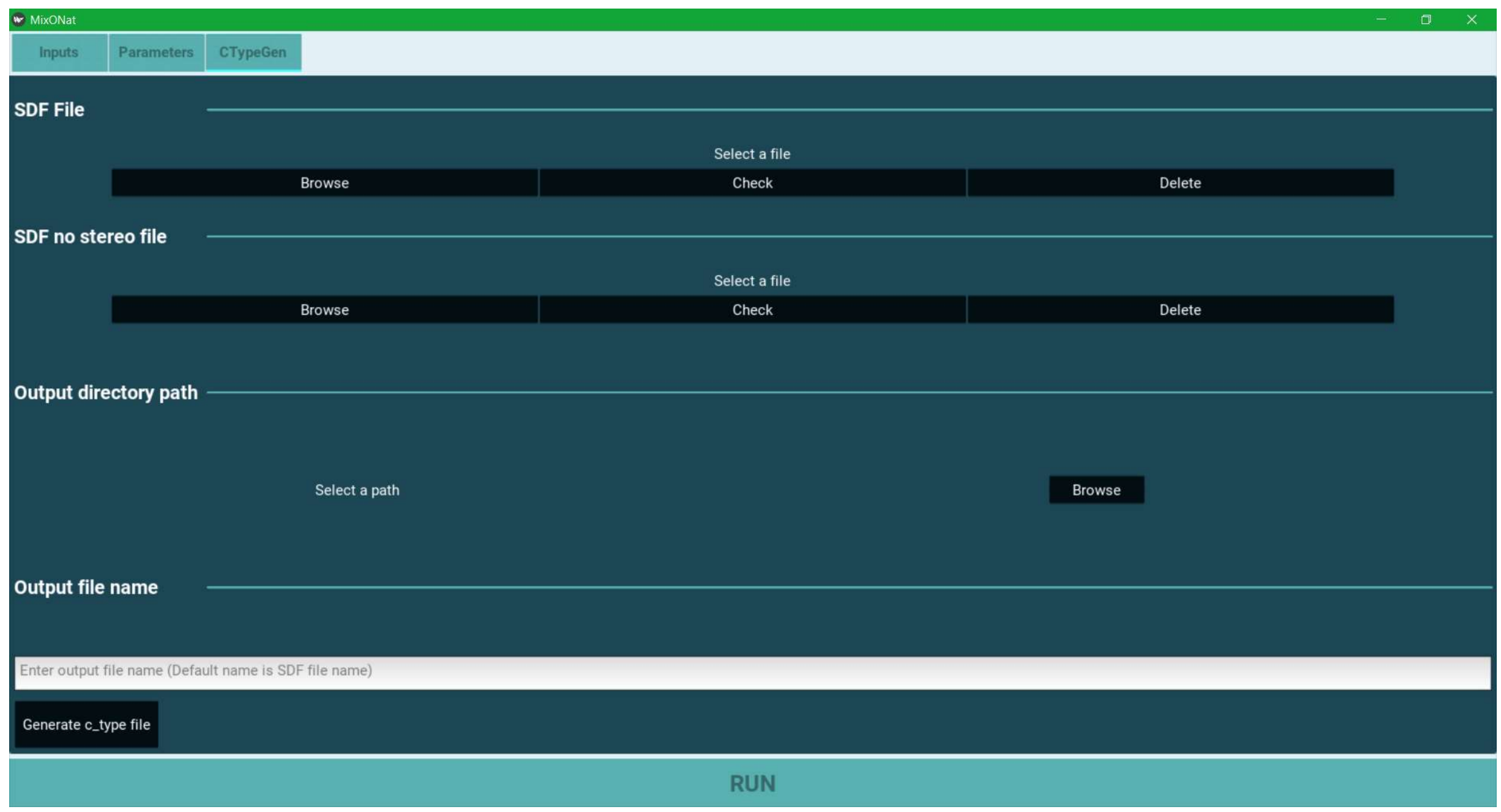

Figure S1. Third tab of the MixONat software corresponding to the C-TypeGen subprogram which sorts chemical shifts according to carbon types. C-TypeGen requires the original DB (.sdf) and the same one without stereochemistry informations. A new .sdf is created with, for each compound of the DB, predicted $\delta_{C}$ values sorted out as methyl, methylene, methine or quaternary carbons. 


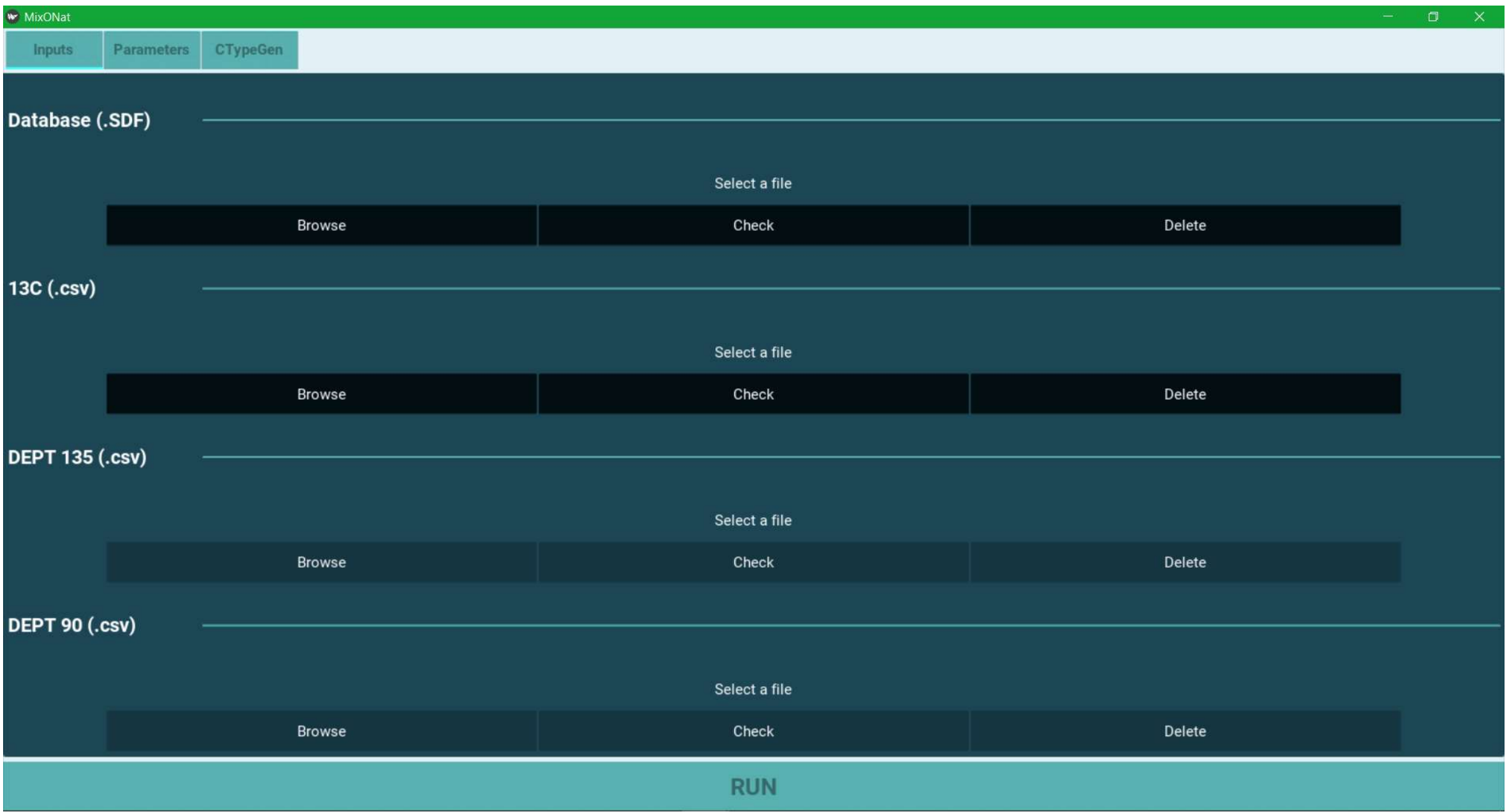

Figure S2. Home tab of the MixONat software. The input files must include at least one database (.sdf) processed by the C-typeGen routine, and ${ }^{13} \mathrm{C}-\mathrm{NMR}$ data, imported as a table (.csv) of $\delta_{\mathrm{c}}$ values and intensities. DEPT-135 and 90 data can also be provided as tables (.csv) of $\delta_{\mathrm{c}}$ values and intensities. 


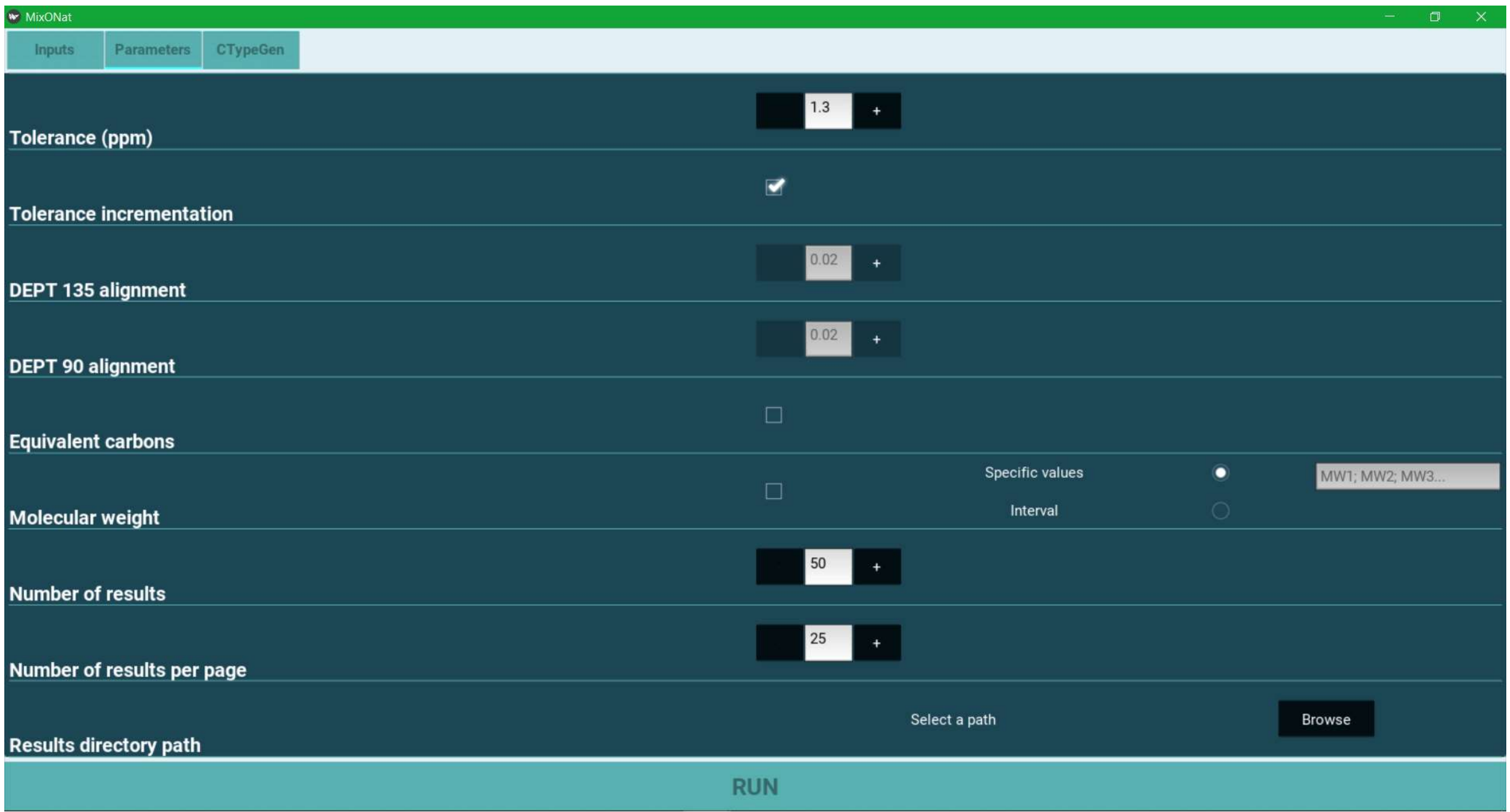

Figure S3. Second tab of the MixONat software. It displays all the different parameters that can be adjusted including the tolerance ( $\varepsilon$ ) (Incrementation ON or OFF), the DEPT alignment parameter; the equivalent carbons option, the molecular weight filter and the minimal score required. The number of results and the directory path for saving results can also be selected in this tab. 


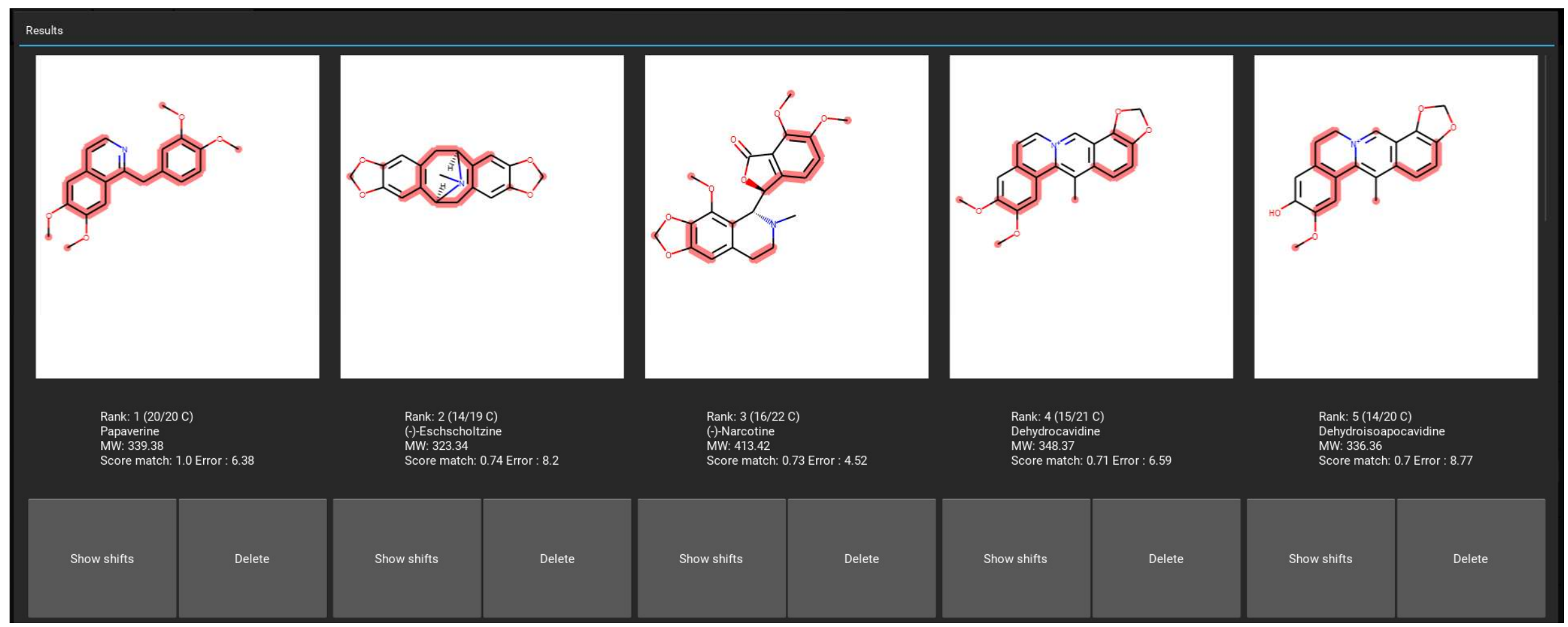

Figure S4. Display of results for the ${ }^{13}$ C-NMR dereplication (+ DEPT 90 and 135) of the poppy extract. Compounds of the DB are ranked by decreasing score and increasing error, with their structure, name, molecular weight, score and error. On the structure, matched carbons are highlighted in red. 


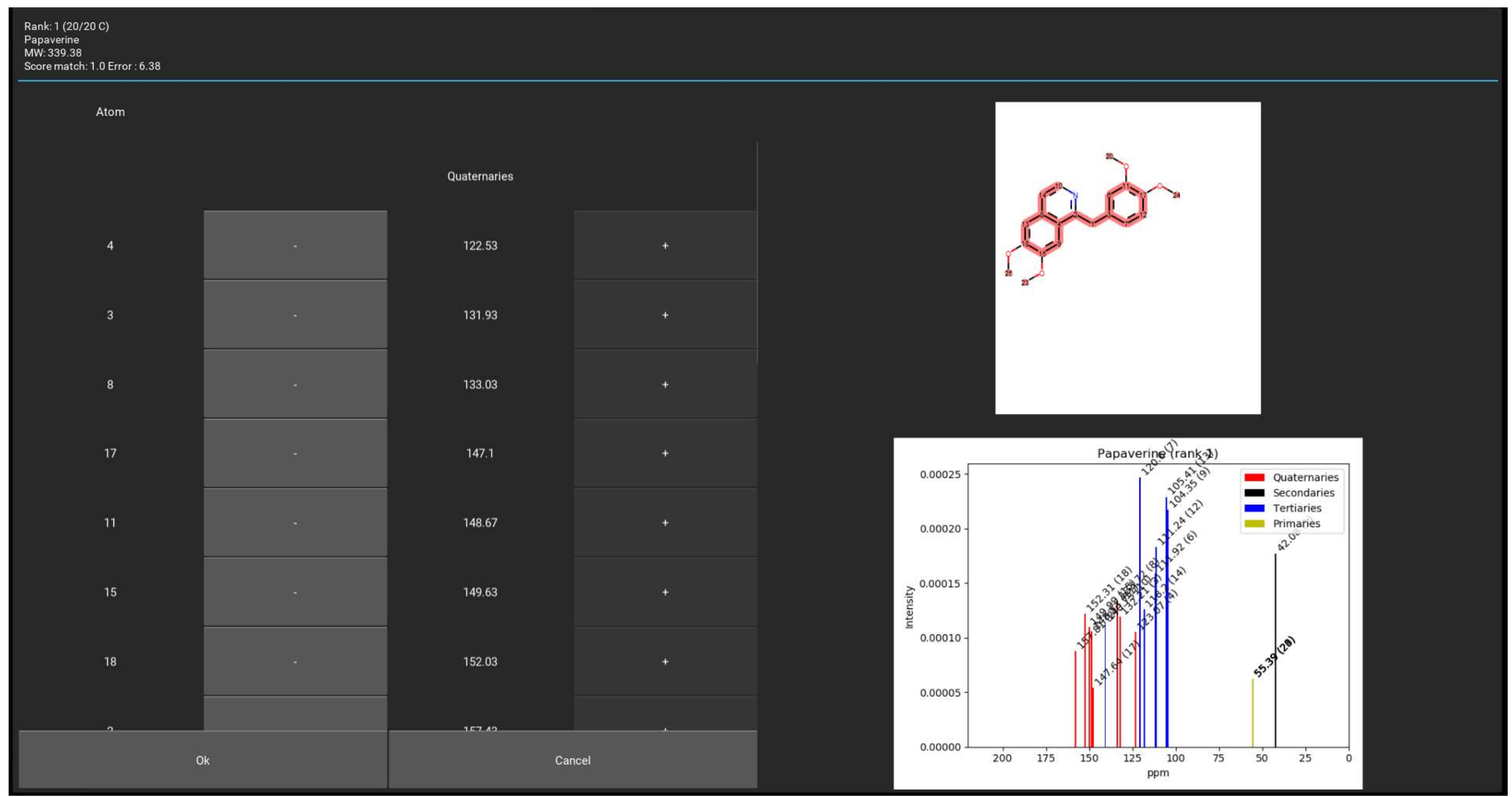

Figure S5. Graphical representation available for each compound (here papaverine from the poppy extract) identified in the DB. A reconstituted ${ }^{13} \mathrm{C}-\mathrm{NMR}$ spectrum shows intensities (with different color types) for matched carbons. 
RESULTS FILE

SDF : c_type_papaveraceae_scif_final.SDF

Spectrum file : papaver_alkaloids.csv

DEPT 135 file : papaver_alkaloids_dept135.cs

DEPT go hle.papaver_alkaloids_dept90.cs

Looseness factor : 1.3

Alignment accuracy $135: 0.05$

gnment accuracy $90: 0.05$

Number of results : 50

Rank: 1

Name : Papaverine
CAS : CAS-58-74-2

MW: 339.38

Score $: 1.0$ (20/20 carbons)

Sectint

Matched spectrum quaternary shifts :

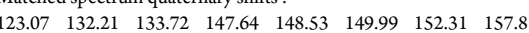

Matched SDF quaternary shifts:

$\begin{array}{llllllll}122.53 & 131.93 & 133.03 & 147.10 & 148.67 & 149.63 & 152.03 & 157.43\end{array}$

Matched quaternary intensities:
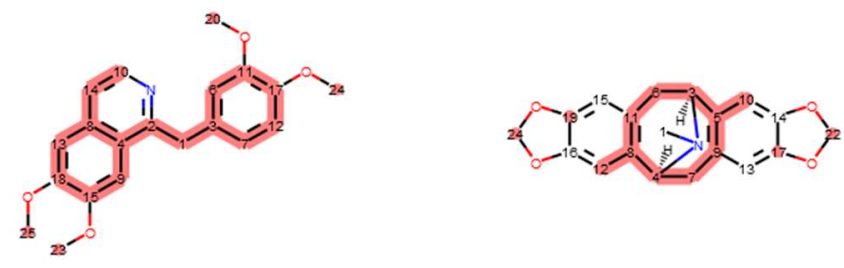

Rank: 1 (20/20 C)

Papaverine

MW: 339.38 match: 10 Error $: 6.38$

Rank: 2 (14/19 C)

(-)-Eschscholtzin

Score match: 0.74 Error : 83

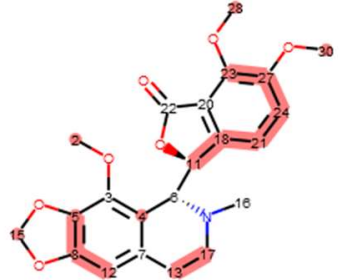

Rank: $3(16 / 22 \mathrm{C})$

MW: 413.42

Score match 0.73 Error $: 4.52$

Matched SDF secondary shifts :

41.97

Matched secondary intensities :

Matched spectrum primary shift

$\begin{array}{llll}55.39 & 55.39 & 55.39 & 55.39\end{array}$

$\begin{array}{lllll}55.50 & 55.50 & 55.50 & 55.50\end{array}$

$\begin{array}{ll}\text { Matched primary intensities: } \\ 0.000062 & 0.000062 \quad 0.000062 \quad 0.000062\end{array}$

Figure S6. Part of displayed results for the ${ }^{13}$ C-NMR dereplication of the poppy extract (+ DEPT 90 and 135$)$ saved as text (Left) and image (Right) files. 
mAU (230 nm)

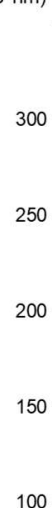

100

50

50

0

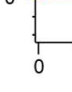

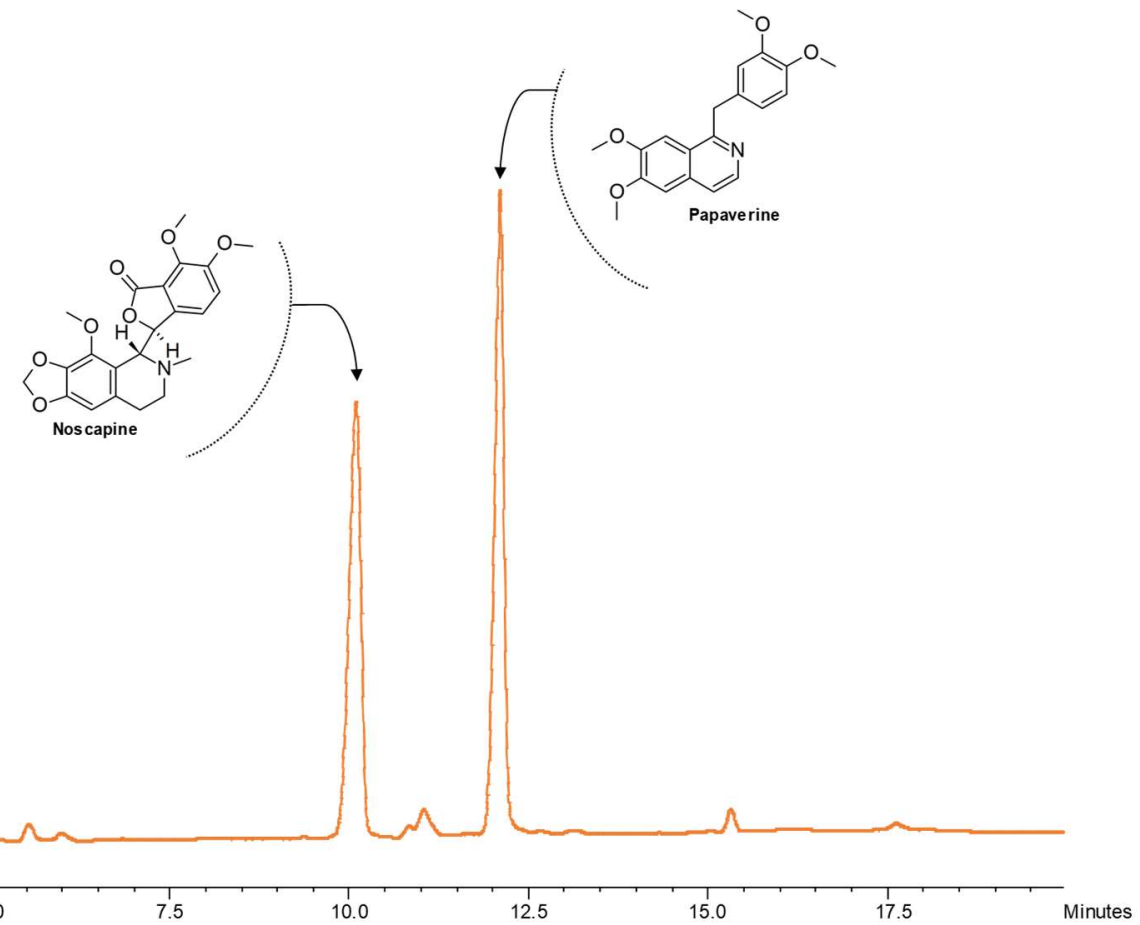

Figure S7. HPLC-UV $(\lambda 230 \mathrm{~nm})$ chromatogram of the Papaver somniferum alkaloids extract 


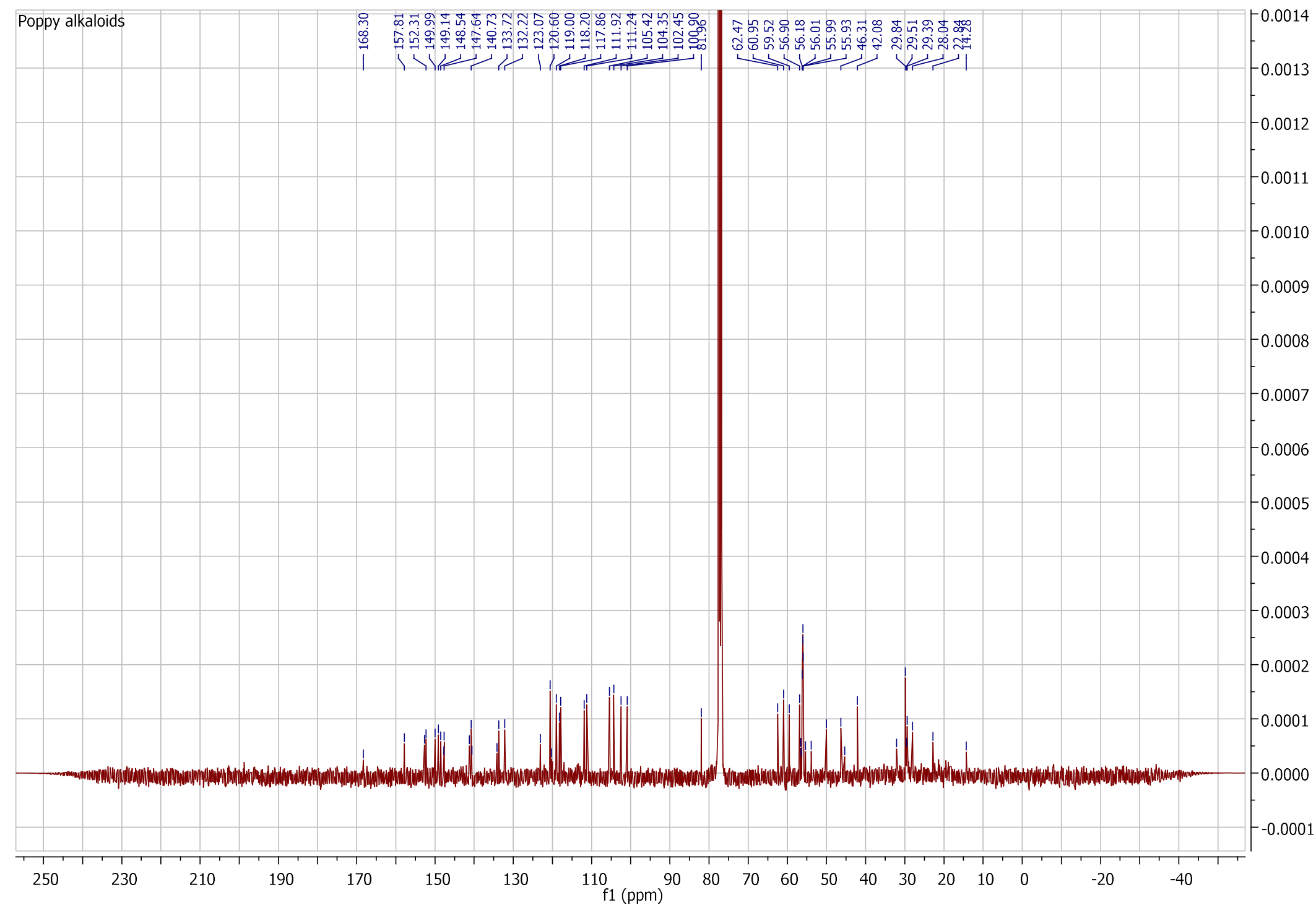

Figure S8. ${ }^{13} \mathrm{C}$-NMR spectrum (17408 scans) of the poppy alkaloids extract ( $\left.10 \mathrm{mg}\right)$ recorded in $\mathrm{CDCl}_{3}$ at $100 \mathrm{MHz}^{-N}$ 
Bruguière et al. Supporting informatio

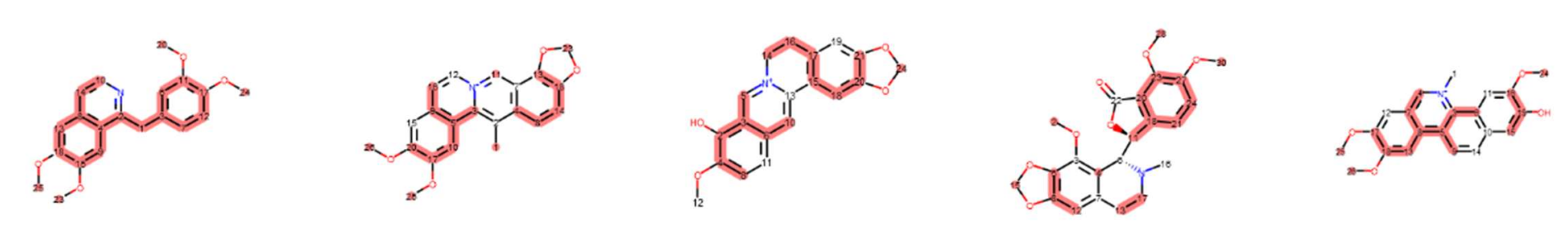

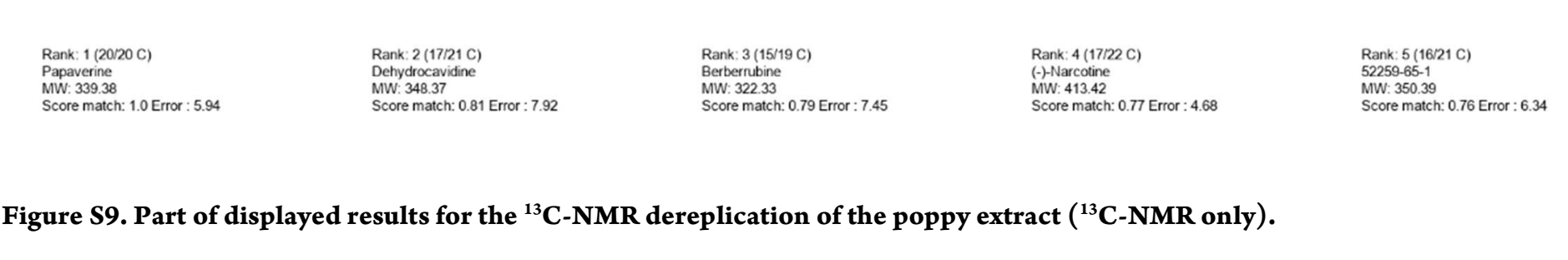

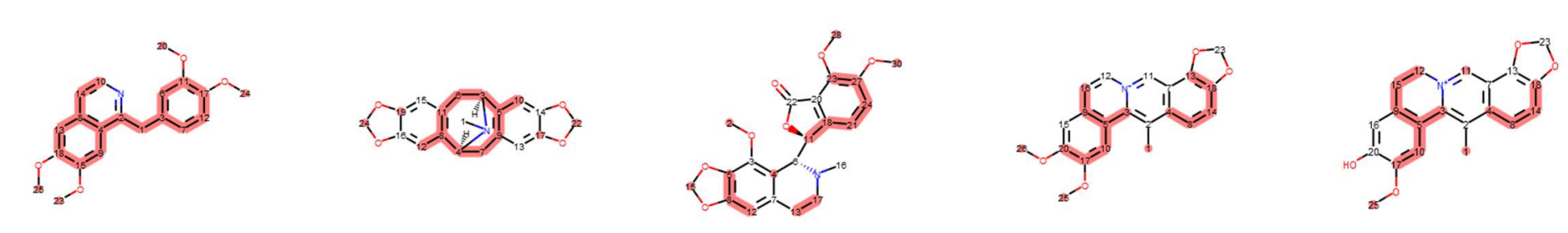

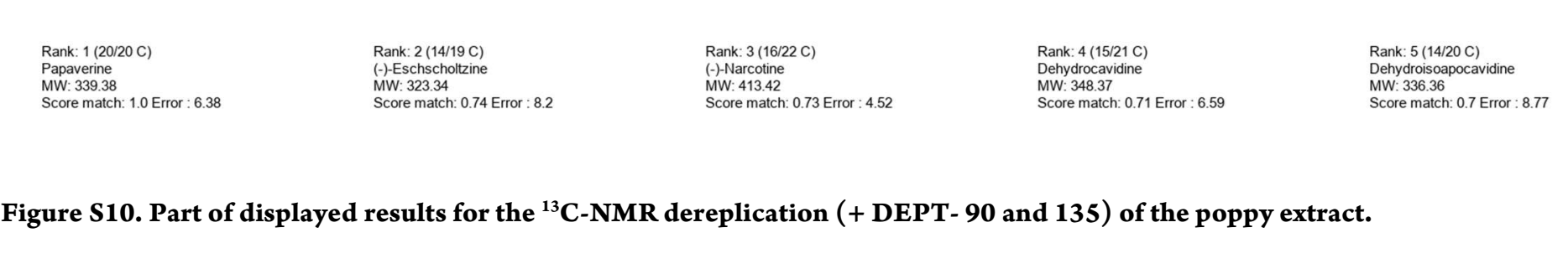


Bruguière et al. Supporting information

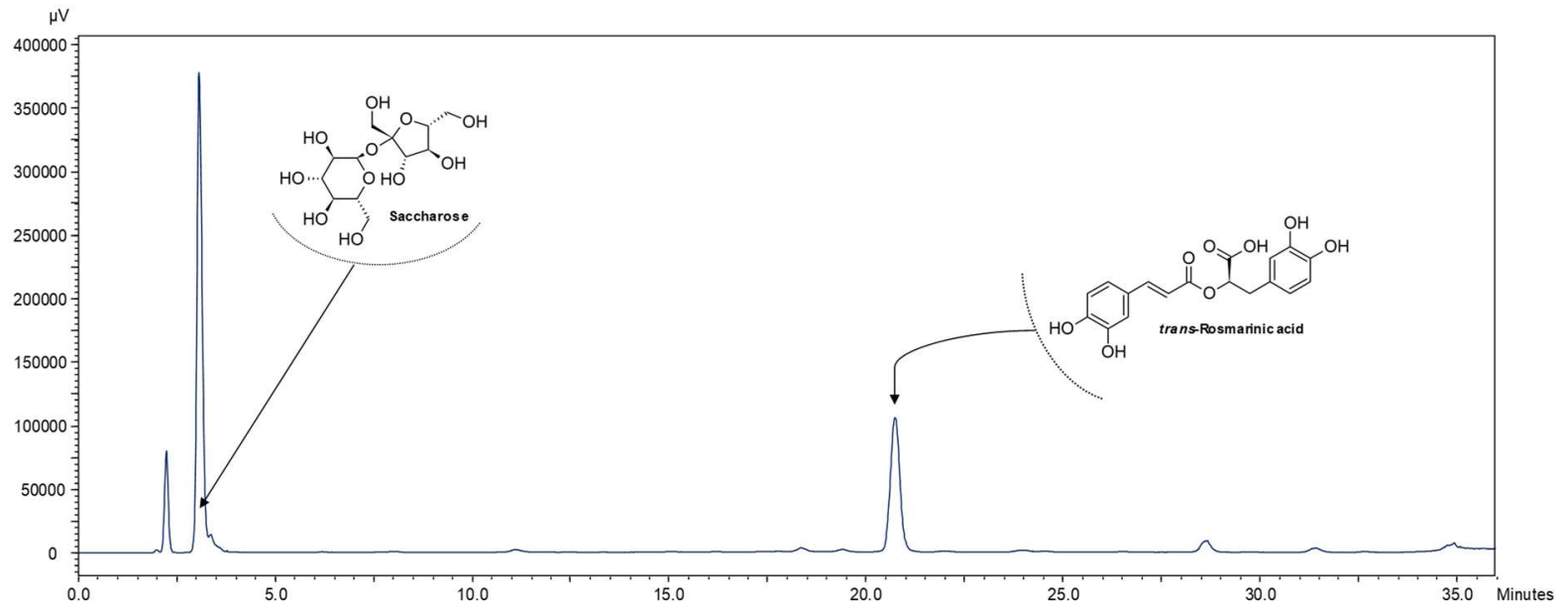

Figure S11. HPLC-ELSD chromatogram of the Rosmarinus officinalis MeOH extract. 
RESULTS FILE

SDF : c_type_LAMIACEAE Final.SDF

Spectrum file : Romarin MeOH.csv

DEPT 135 file : Romarin $\mathrm{MeOH}$ dept135.csv

DEPT 90 file : Romarin MeOH_dept90.cs

Looseness factor : 1.3

Alignment accuracy $135: 0.02$

Alignment accuracy $90: 0.02$

Number of results : 25

Equivalent carbons not allowed

Rank: 4
ID : 969

Name : trans-Rosmarinic acid

CAS: CAS-20283-92-5

$\mathrm{MW}: 360.31$

Score $: 0.83$ (15/18 carbons)

Cumulated absolute difference : 3.2

Spectrum shifts left: $14 / 29$

Matched spectrum quaternary shifts :

$\begin{array}{llllll}127.82 & 144.88 & 145.95 & 14670 & 149.43 & 168.92\end{array}$

Matched SDF quaternary shifts:
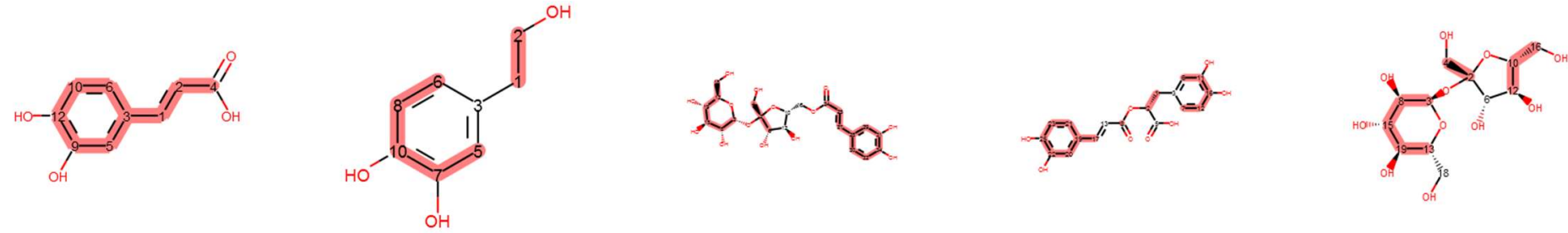

Rank: $1(9 / 9 \mathrm{C})$

Cafteic acid
MW: 180.16
Score match: 1.0 Error : 3.97

Rank: 2 (778 C)

3.-Hydroxytyrosol
MW: 154.16
Score match. 088 Error $: 26$

Rank: $3(18 / 21$ C)
CAS-1345877-80-6

CAS-1345877-80-6
MW 504.44
Score match: 086 Error 4 - 11

Rank: 4 (15/18 C)

trans-Rosmarini

Rank: $5(10 / 12 \mathrm{C})$

D-(t)-Sucrose
MW: 342.3

Score match: 0.83 Error : 5.83

$\begin{array}{llllll}127.70 & 145.08 & 145.96 & 146.80 & 149.70 & 168.50\end{array}$

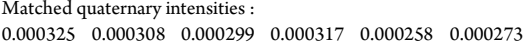

$129.29 \quad 173.76$

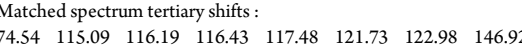

Matched SDF tertiary shifts :

$\begin{array}{llllllll}75.00 & 115.10 & 116.25 & 116.50 & 117.54 & 121.74 & 123.10 & 147.64\end{array}$

Mathed tertianlitensties:

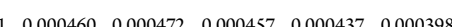

Not matched SDF tertiary shifts

117.07

Matched spectrum secondary shifts :

Matched SDF secondary shifts :

Matched

Figure S12. Part of displayed results for the ${ }^{13} \mathrm{C}-\mathrm{NMR}$ dereplication (+ DEPT 90 and 135) of the rosemary MeOH extract. Equivalent carbons were allowed. 
Bruguière et al. Supporting information

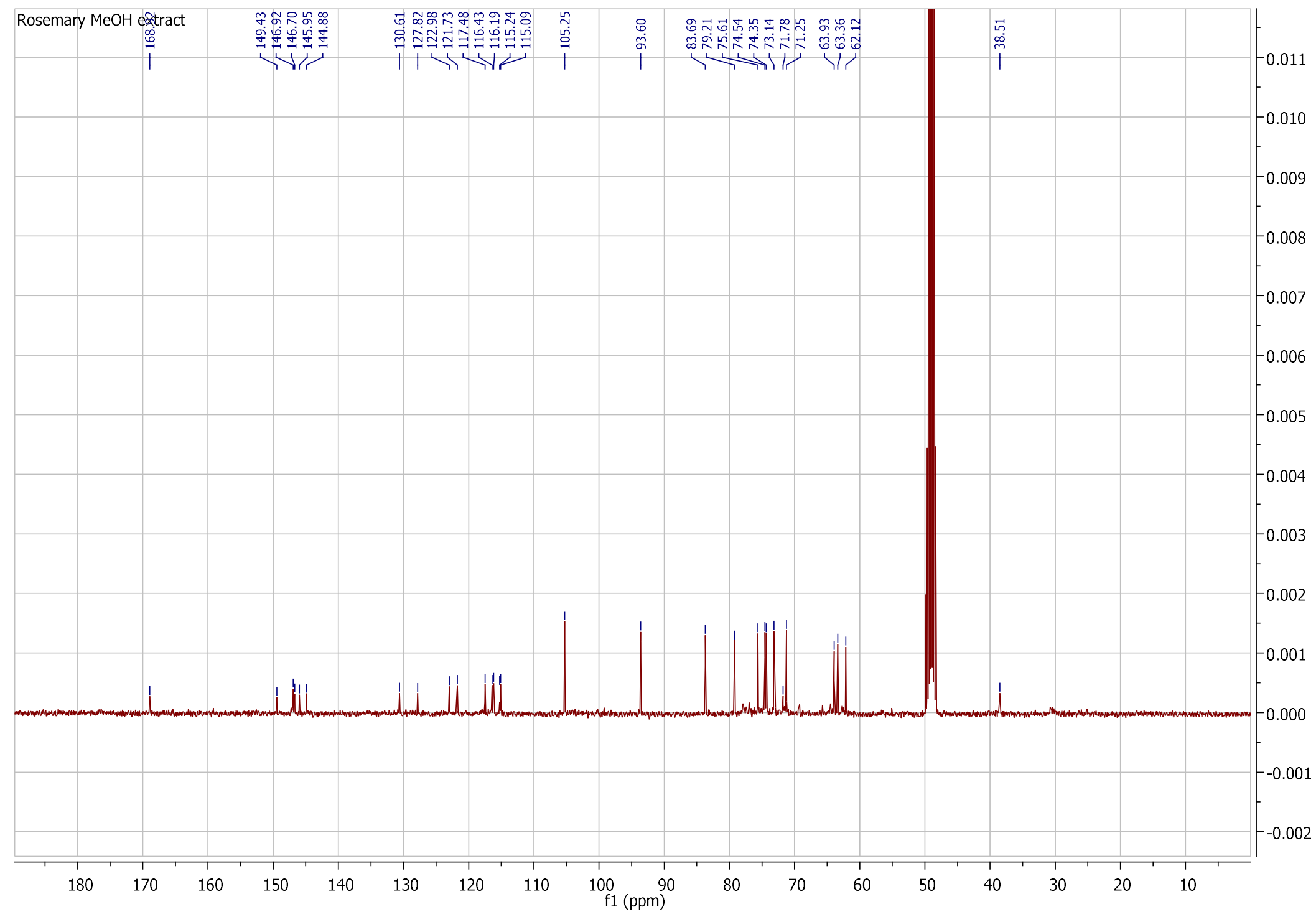

Figure S13. ${ }^{13} \mathrm{C}$-NMR spectrum (2048 scans) of the rosemary leaf $\mathrm{MeOH}$ extract (50 $\mathrm{mg}$ ) recorded in methanol-d $\mathrm{d}_{4}$ at $100 \mathrm{MHz}$ 


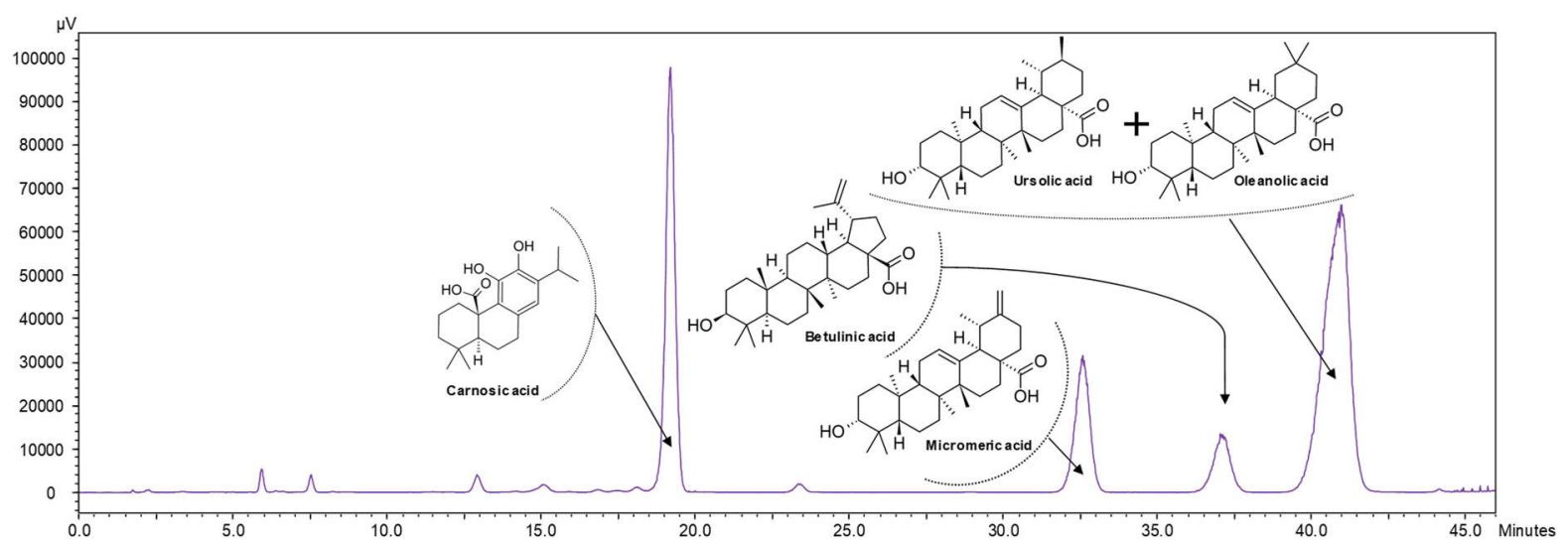

Figure S14. HPLC-ELSD chromatogram of the E392 extract.

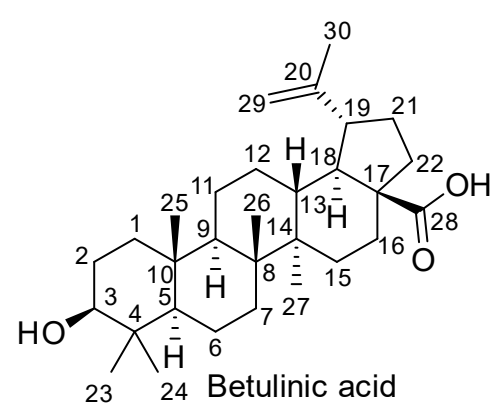

Micromeric acid
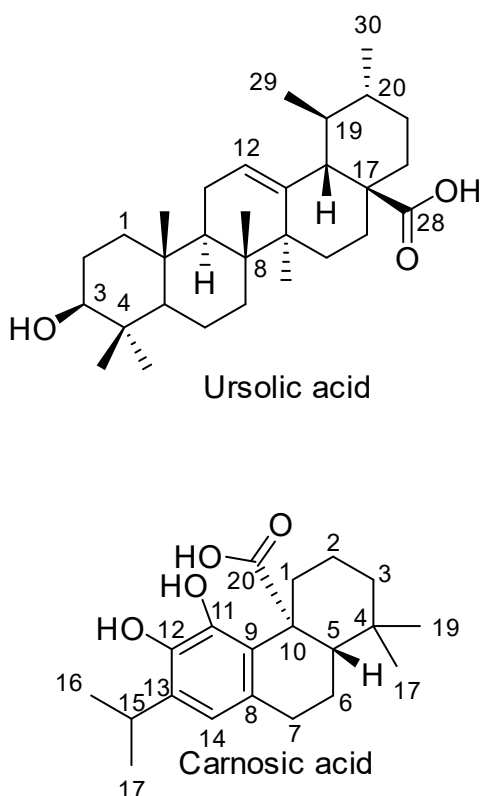

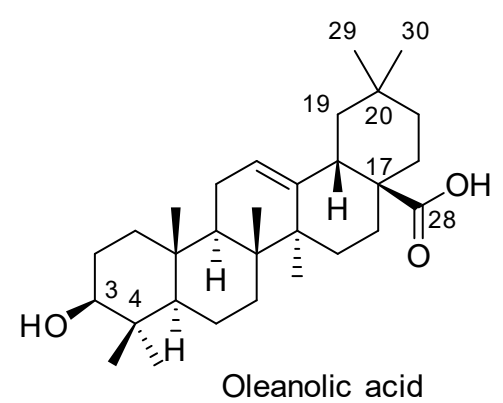

Oleanolic acid

Figure S15. Structures of terpenes from E392 extract 

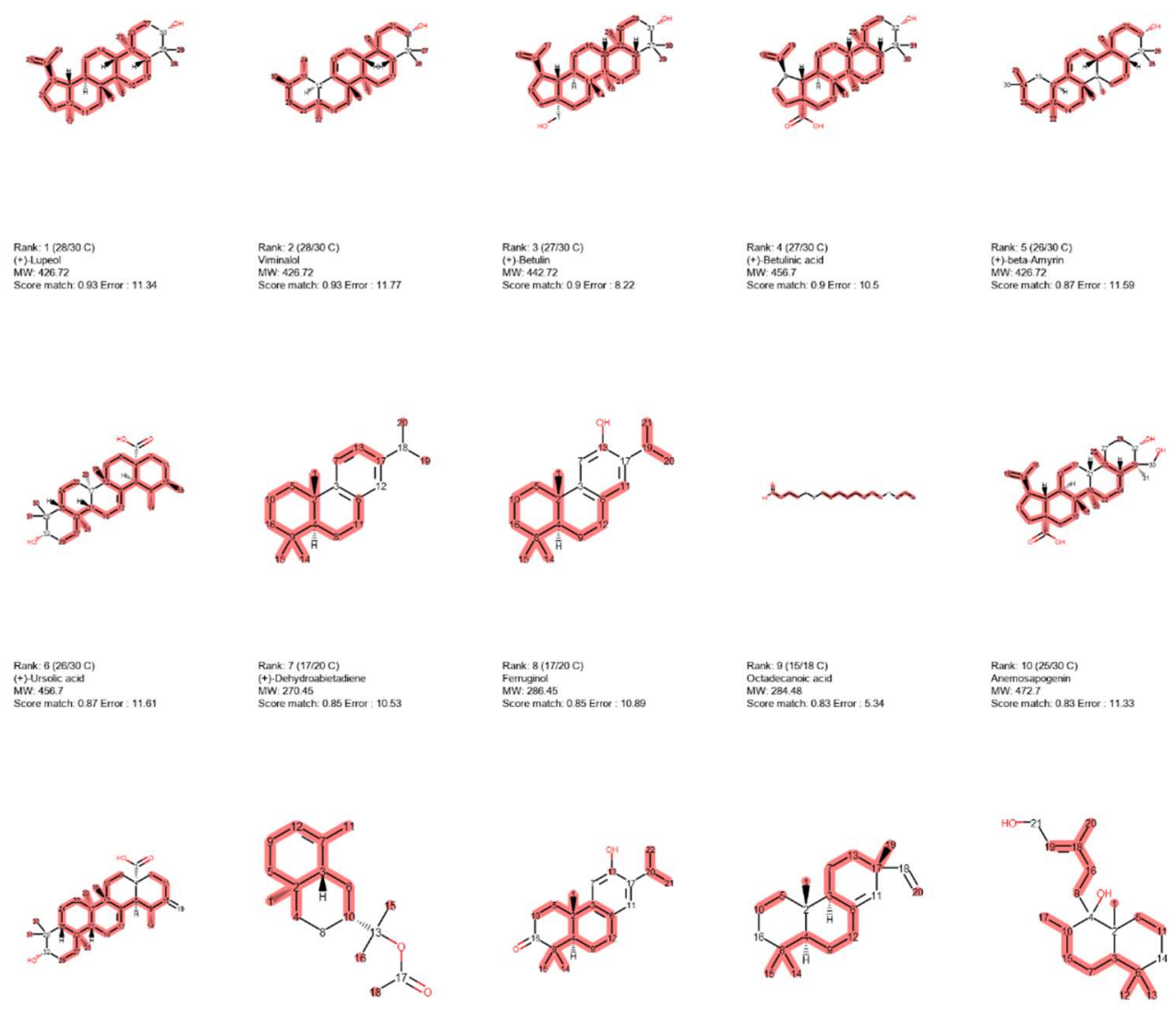

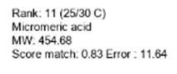
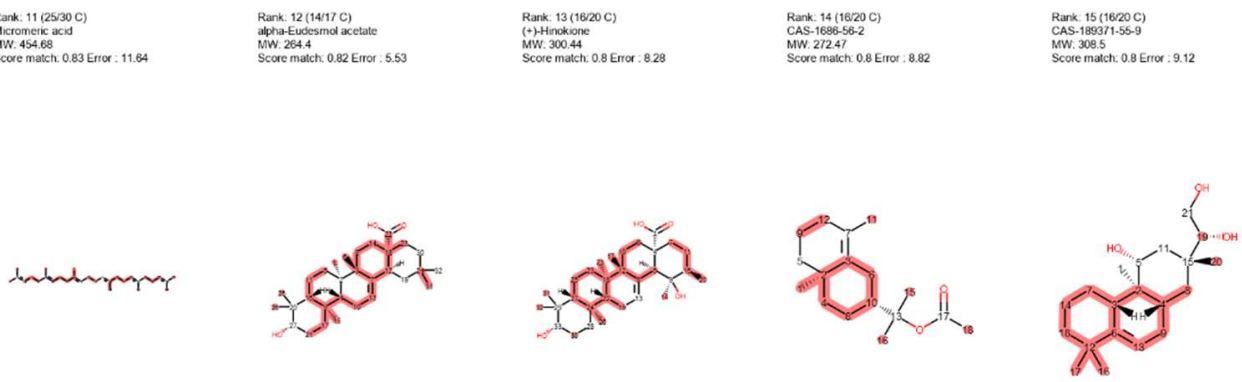

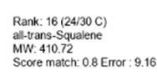

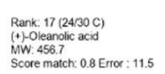
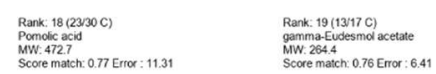

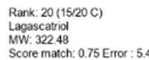
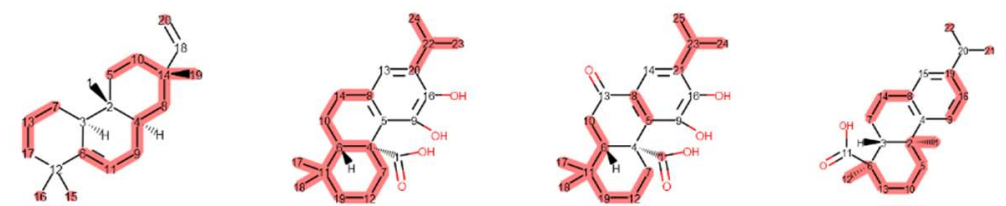

Rank 21 (15:20 C)
Rimuene

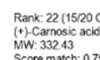

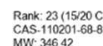

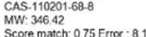

Rank: $24(1520 \mathrm{C})$
CAS $1470.19-8$
MW: 300.44

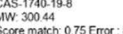

Figure S16. Part of displayed results for the ${ }^{13}$ C-NMR dereplication (+ DEPT 90 and 135) of the rosemary E392 extract. Equivalent carbons were allowed. A molecular weight filter ( $250 \mathrm{Da}$ ) was applied. 


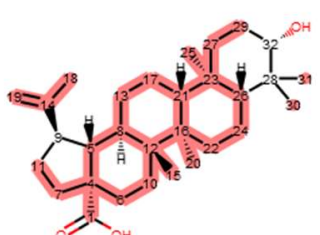

Rank: $1(27 / 30 \mathrm{C})$

MW: 456.7

Score match: 0.9 Error : 10.5
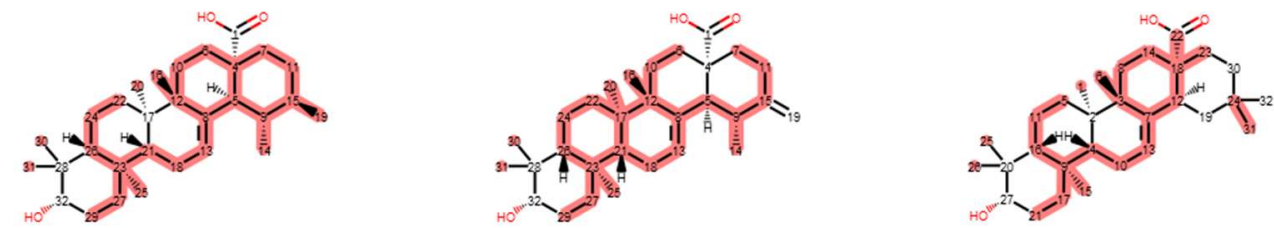

Rank: 4 (24/30 C)

Rank: 3 (25/30 C)

Rank: $2(26 / 30 \mathrm{C})$

(+)-Ursolic acid
MW: 456.7

Score match: 0.87 Error : 11.61
Micromeric acid

Score match: 0.83 Error : 11.64

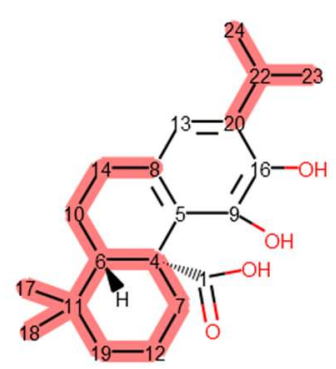

Rank: 5 (15/20 C) (+)-Carnosic acid

Score match: 0.75 Error : 6.63

Figure S17. Part of displayed results for the ${ }^{13}$ C-NMR dereplication (+ DEPT 90 and 135) of the rosemary E392 extract. Equivalent carbons were allowed. Only NPs with MW 330, 332, 454 or 456 Da were allowed. 


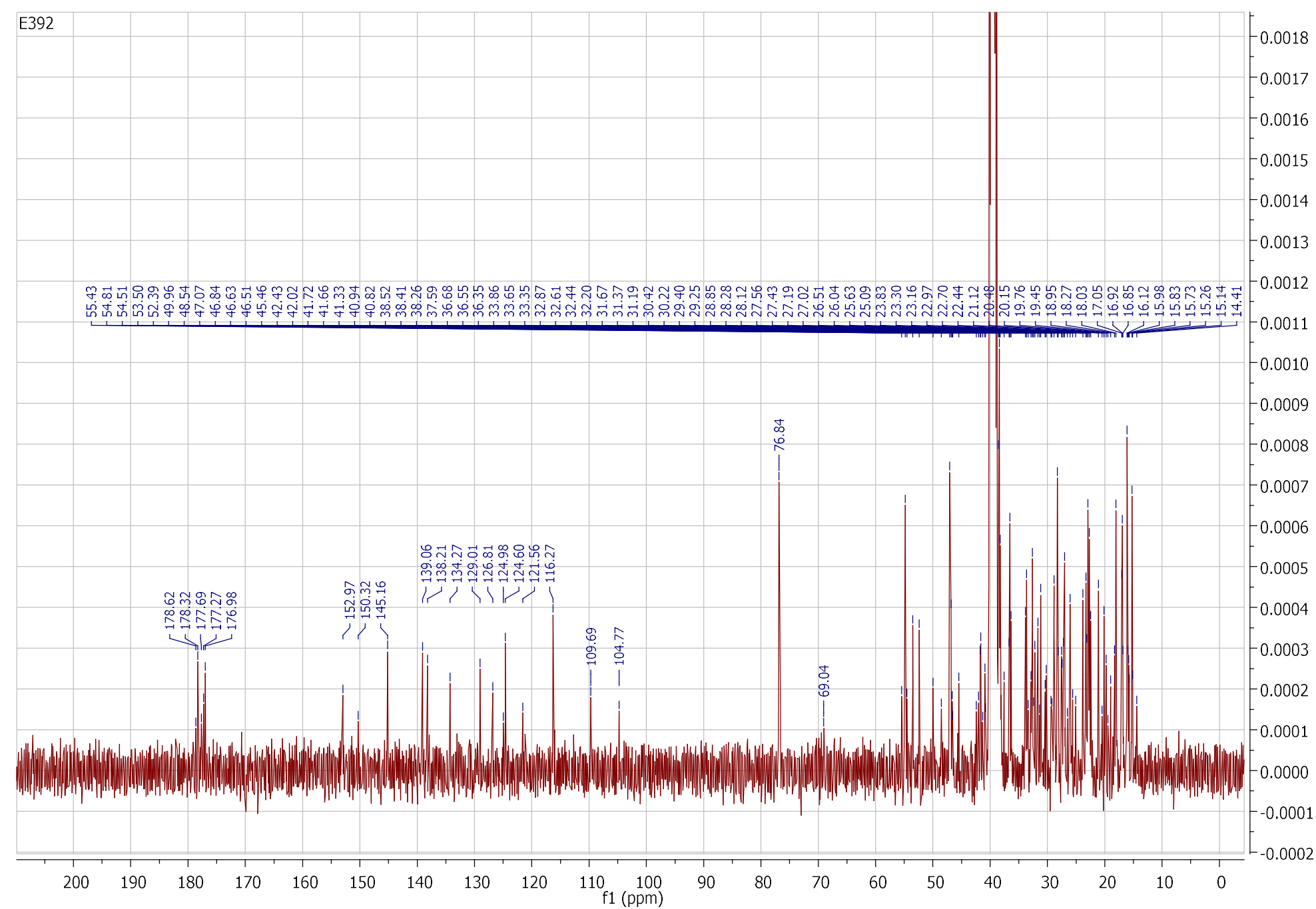

Figure S18. ${ }^{13} \mathrm{C}-\mathrm{NMR}$ spectrum (1024 scans) of the rosemary E392 extract ( $50 \mathrm{mg}$ ) recorded in DMSO-d6 at $100 \mathrm{MHz}$ 
Bruguière et al. Supporting information

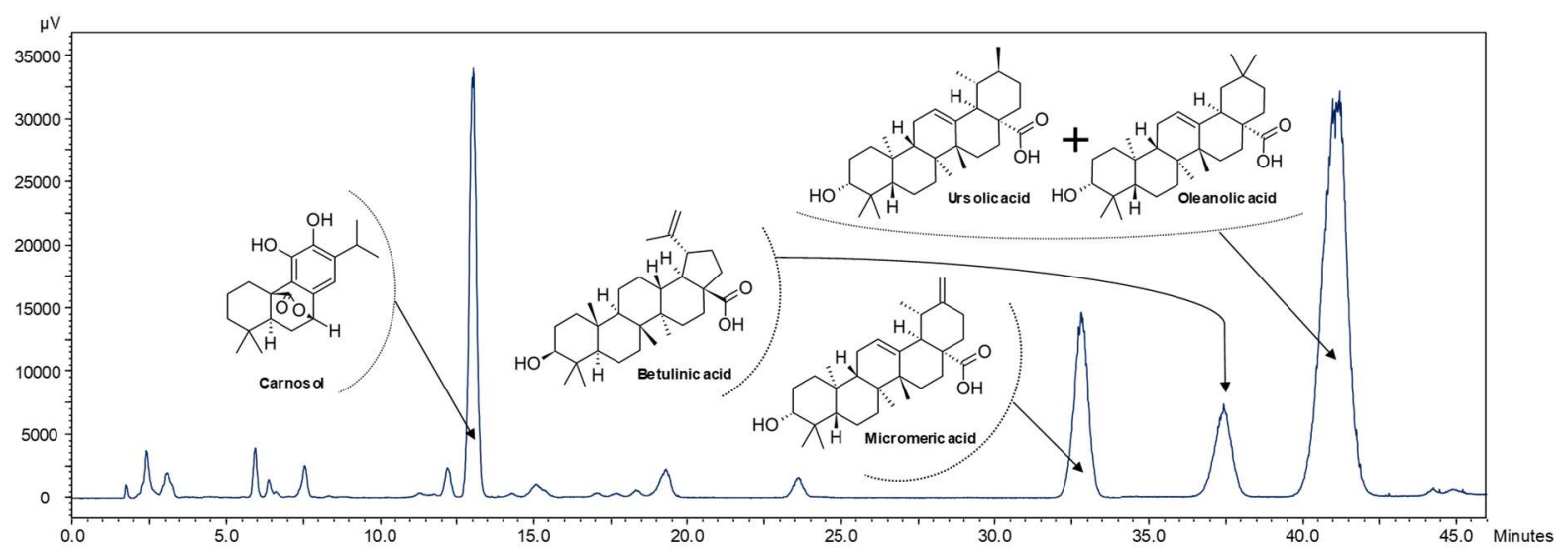

Figure S19. HPLC-ELSD chromatogram of the Rosmarinus officinalis DCM extract. 


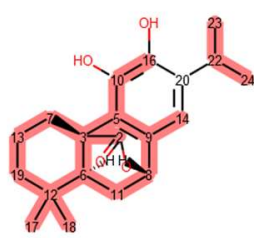

Rank: 1 (19/20 C)
Carnosol

Carnosol
MW: 30.42
Scoremath

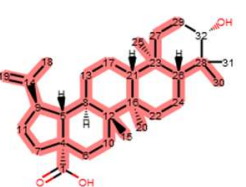

Rank: 2 (288130 C)
(+)-Betulinic acid
MW: 456.7

Rank: $3(27 / 30 \mathrm{C})$
(+)-Ursolic acid

MW: 456.7 .

Rank: 4 (26/30 C)
Micromeric acid
MW: 454.68

Micromeric acid
MW: 454.68
Score math

Rank: $5(26 / 30 \mathrm{C})$
$(+)-$ - leanolic acid

MW: 456.7 .

Rank: $6(17720 \mathrm{C})$
$(+)$-Carnosic acid

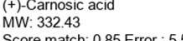

Figure S20. Part of displayed results for the ${ }^{13}$ C-NMR dereplication (+ DEPT 90 and 135) of the rosemary DCM extract. Equivalent carbons were allowed Only NPs with MW 330, 332, 454 or 456 Da were allowed. 


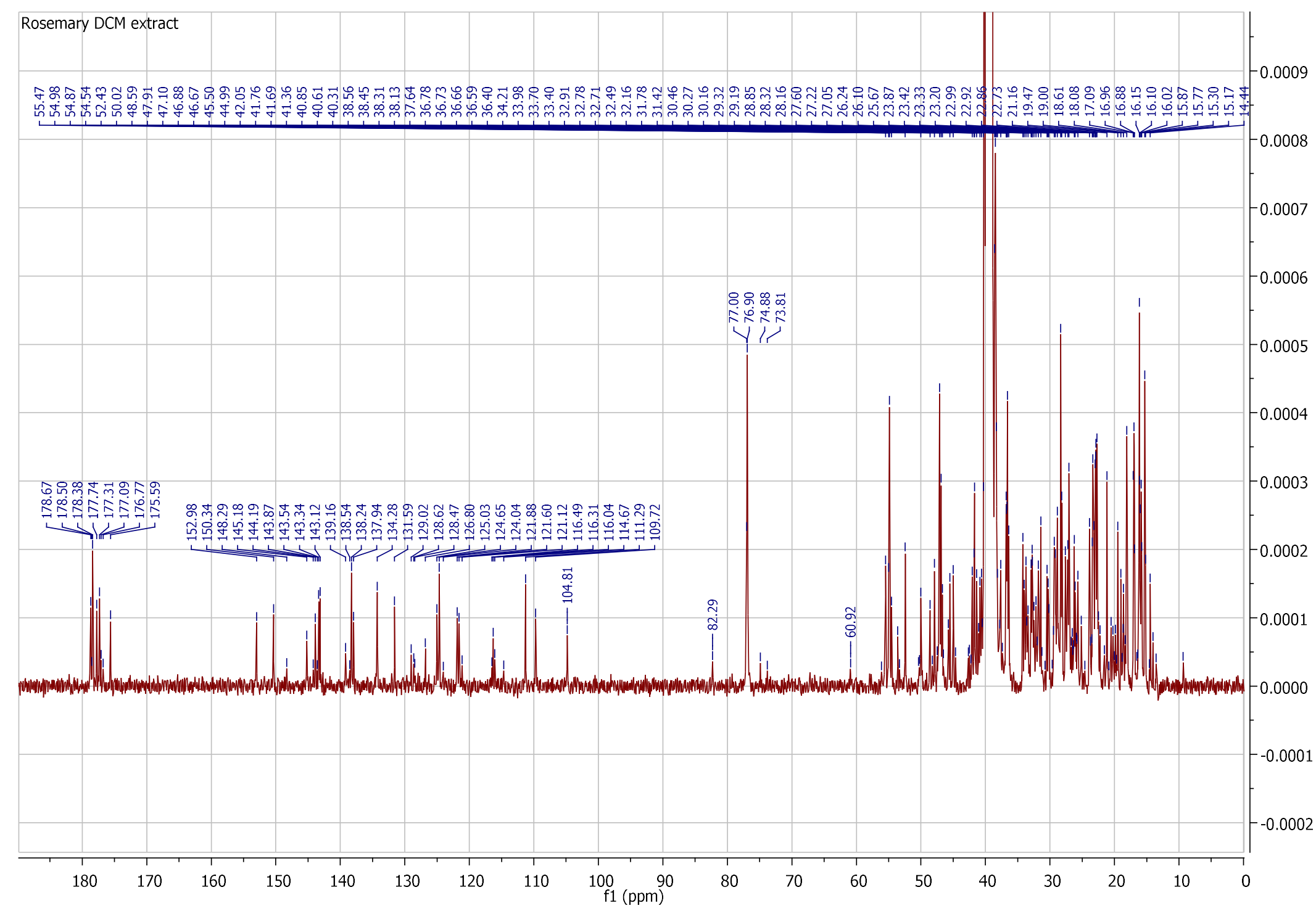

Figure S21. ${ }^{13} \mathrm{C}-\mathrm{NMR}$ spectrum (10000 scans) of the rosemary leaf DCM extract $(50 \mathrm{mg})$ recorded in DMSO-d6 at $100 \mathrm{MHz}$ 
A.

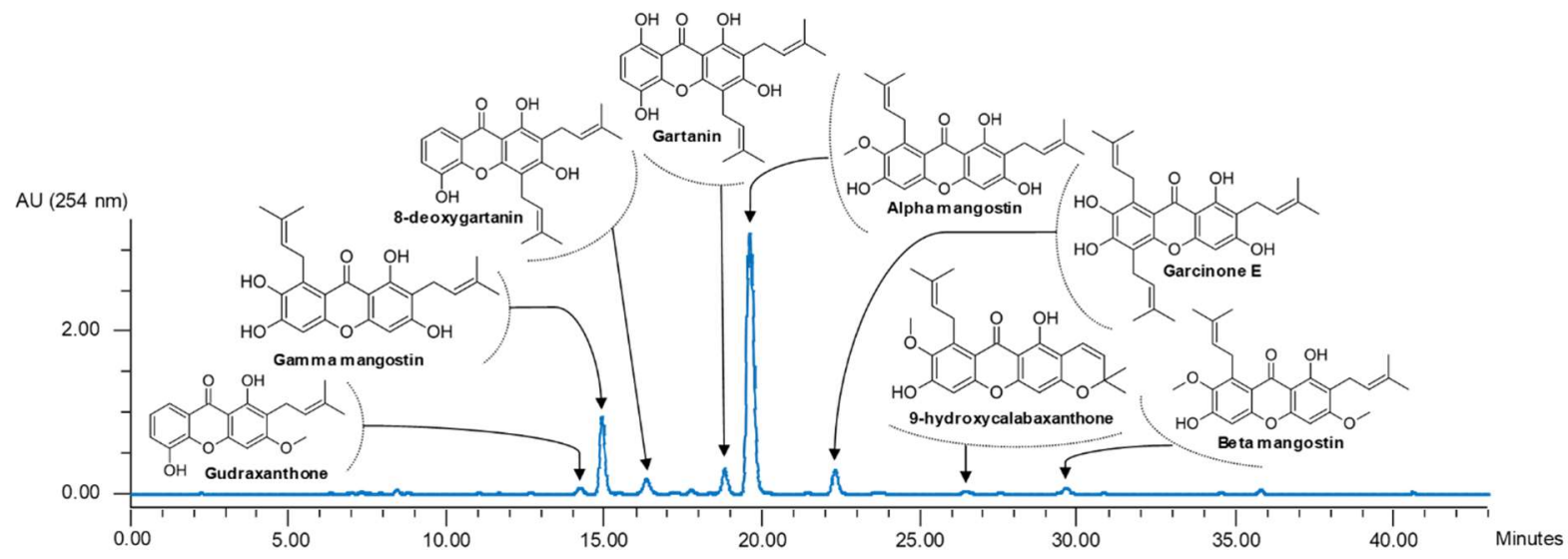

B.

\begin{tabular}{|ccc|}
\hline Name & Retention time (min) & Area (\%) \\
\hline $\boldsymbol{\alpha}$-mangostin & 19.6 & 68 \\
\hline $\boldsymbol{\gamma}$-mangostin & 14.9 & 14 \\
\hline Gartanin & 18.8 & 5 \\
\hline Garcinone E & 22.2 & 5.5 \\
\hline 8-desoxygartanin & 16.3 & 1.5 \\
\hline 及-mangostin & 29.6 & 1.0 \\
\hline Gudraxanthone & 14.2 & $<1 \%$ \\
\hline 9-hydroxycalabaxanthone & 26.4 & \\
\hline
\end{tabular}

C.<smiles>[R20]Oc1cc2oc3c([R2])c(O)c(CC=C(C)C)c(CC=C(C)C)c3c(=O)c2c(O)c1CC=C(C)C</smiles>

$\alpha$-mangostin: $\mathrm{R}_{1}=\mathrm{H}, \mathrm{R}_{2}=\mathrm{H}, \mathrm{R}_{3}=\mathrm{Me}$ $\beta$-mangostin: $\mathrm{R}_{1}=\mathrm{Me}, \mathrm{R}_{2}=\mathrm{H}, \mathrm{R}_{3}=\mathrm{Me}$ $\gamma$-mangostin: $\mathrm{R}_{1}=\mathrm{H}, \mathrm{R}_{2}=\mathrm{H}, \mathrm{R}_{3}=\mathrm{H}$ Garcinone $E: R_{1}=H, R_{2}=$ prenyl, $R_{3}=H$<smiles>[R2]c1c(O)c([R6])[13c](CC=C(C)C)c2c(=O)c3c(O)c4c(cc3oc12)OC(C)(C)C=C4</smiles>

9-hydroxycalabaxanthone: $\mathrm{R}_{2}=\mathrm{H}, \mathrm{R}_{3}=\mathrm{Me}$<smiles>[R]Oc1c(CC=C(C)C)c(O)c2c(=O)c3[Z]([R5])ccc(O)c3oc2c1[R4]</smiles>

Gartanin: $\mathrm{R}_{1}=\mathrm{H}, \mathrm{R}_{4}=$ prenyl, $\mathrm{R}_{5}=\mathrm{OH}$ 8-deoxygartanin: $R_{1}=H, R_{4}=$ prenyl, $R_{5}=H$ Gudraxanthone: $\mathrm{R}_{1}=\mathrm{Me}, \mathrm{R}_{4}=\mathrm{H}, \mathrm{R}_{5}=\mathrm{H}$

Figure S22. (A) HPLC-UV ( $\lambda 254 \mathrm{~nm}$ ) chromatogram of the Garcina mangostana (fruit peel) cyclohexanic extract (B) \% (area) of each xanthone peak. (C) Structures of $G$. mangostana xanthones 

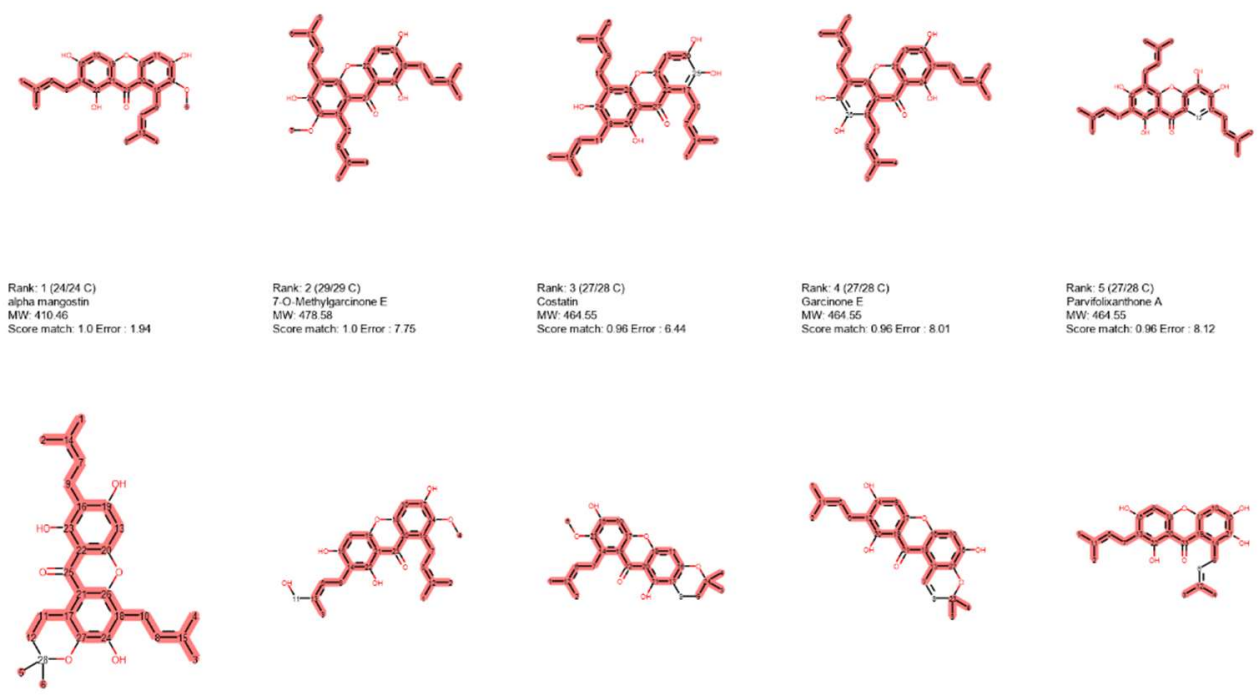

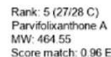
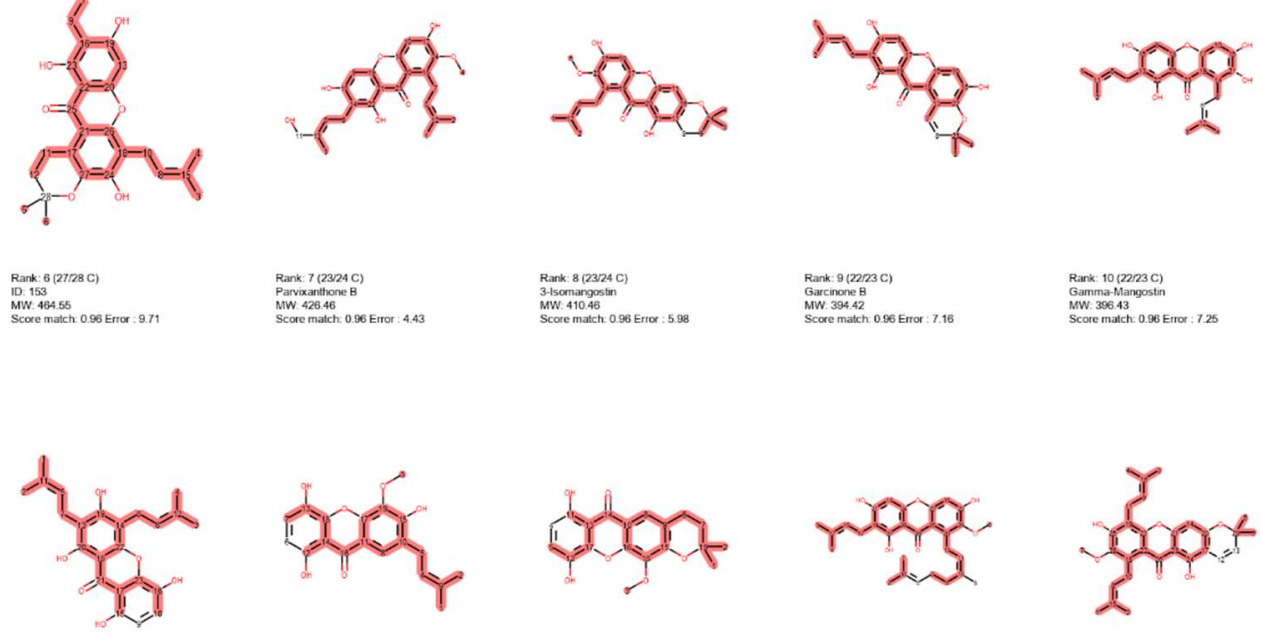

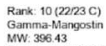

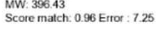

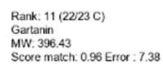
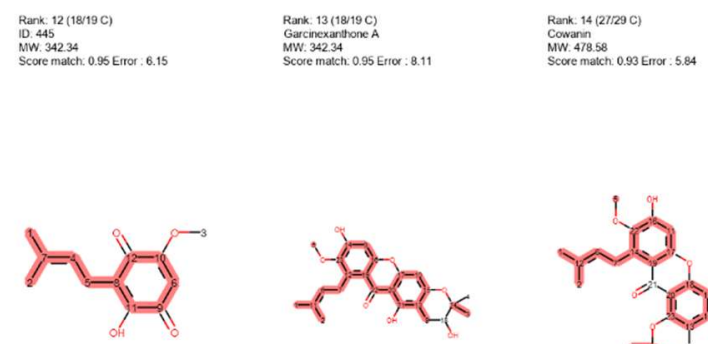

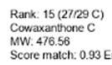

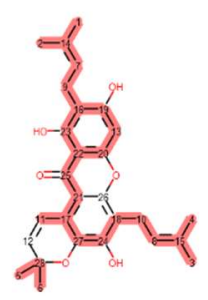

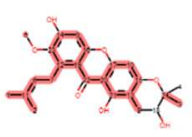

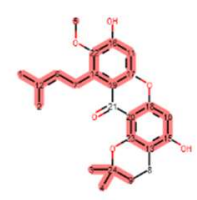

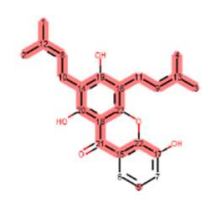

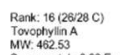<smiles>C#CCCC(C)CC(C)C</smiles>

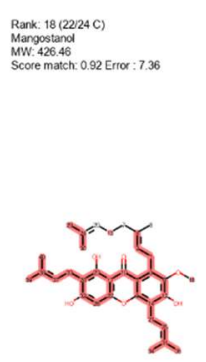

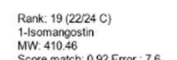

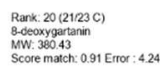

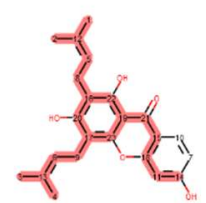

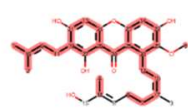

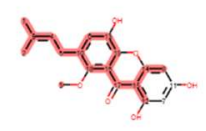

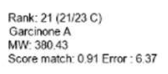

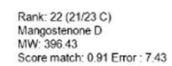

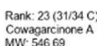

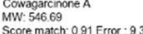

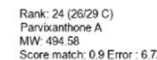

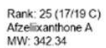

Figure S23. Part of displayed results for the ${ }^{13}$ C-NMR dereplication (+ DEPT 90 and 135) of the mangosteen (fruit peel) cyclohexanic extract. Equivalent carbons were allowed. 


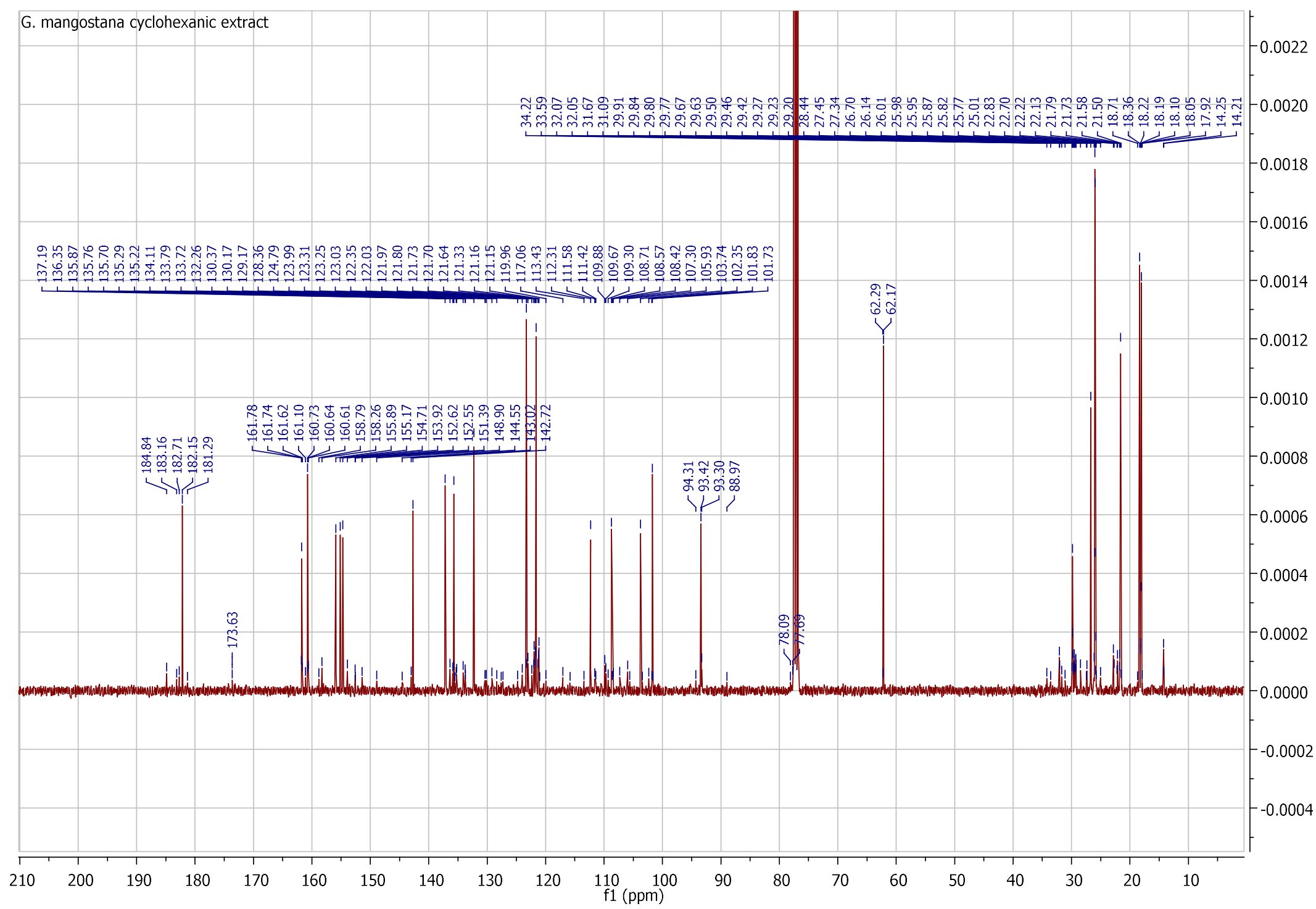

Figure S24. ${ }^{13} \mathrm{C}-\mathrm{NMR}$ spectrum (10000 scans) of the mangosteen (fruit peel) cyclohexanic extract (30 $\left.\mathrm{mg}\right)$ recorded in $\mathrm{CDCl}_{3}$ at $100 \mathrm{MHz}^{-}$ 


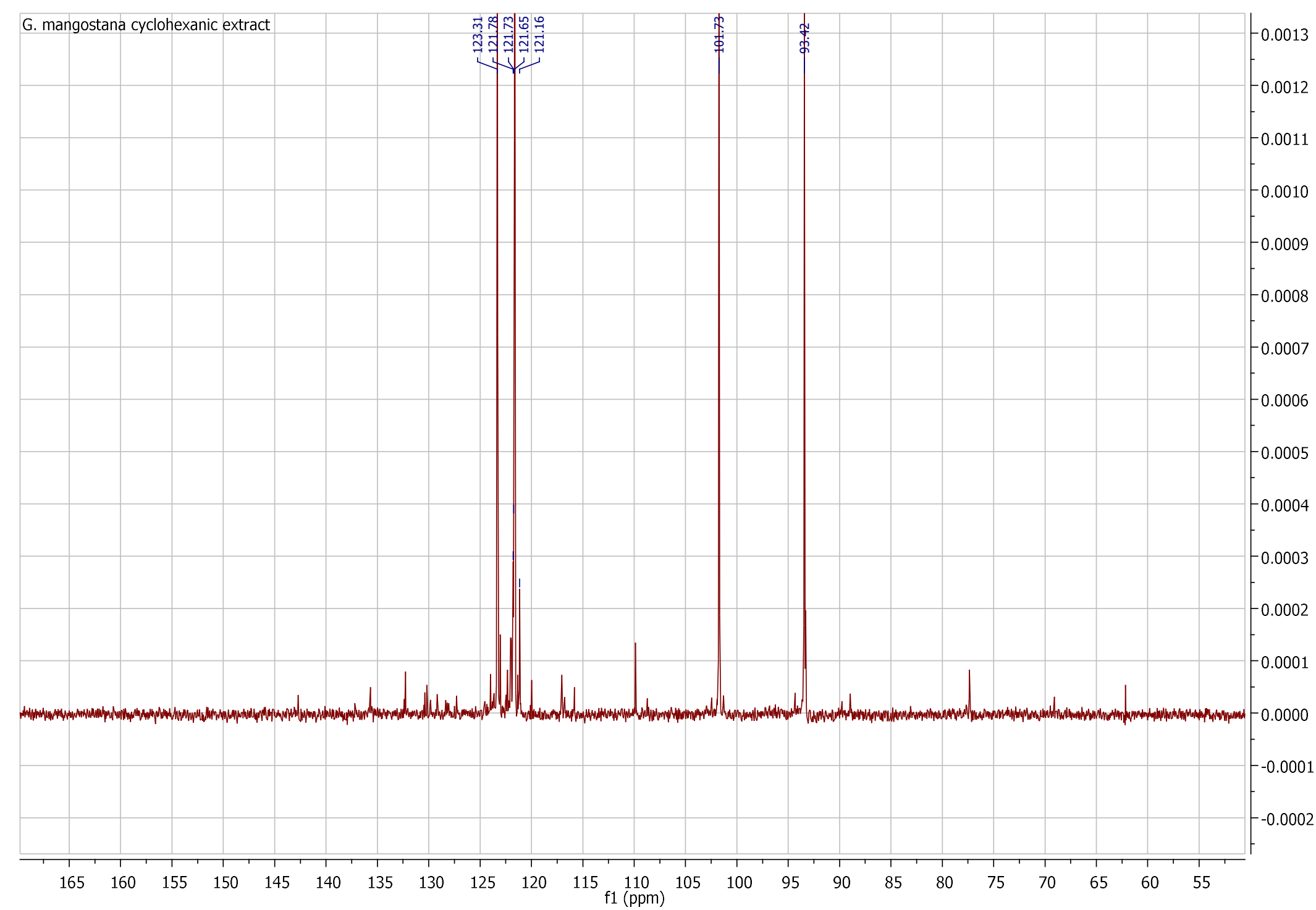

Figure S25. DEPT-90 spectrum (5000 scans) of the mangosteen fruit peel cyclohexanic extract ( $30 \mathrm{mg})$ recorded in $\mathrm{CDCl}_{3}$ at $100 \mathrm{MHz}$ 


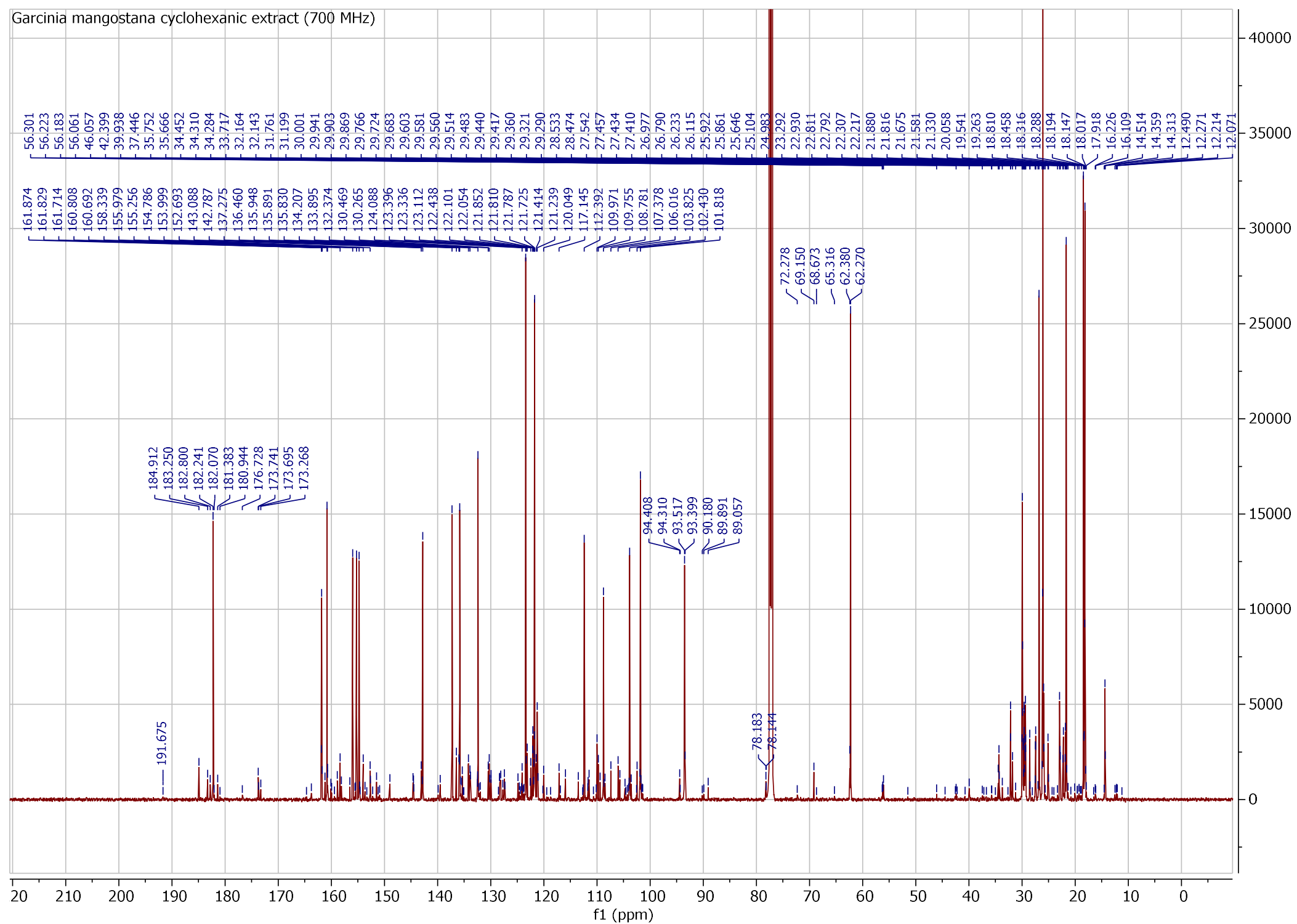

Figure S26. ${ }^{13} \mathrm{C}$-NMR spectrum (10000 scans) of the mangosteen (fruit peel) cyclohexanic extract (30 $\left.\mathrm{mg}\right)$ recorded in $\mathrm{CDCl}_{3}$ at $175 \mathrm{MHz}^{-}$ 

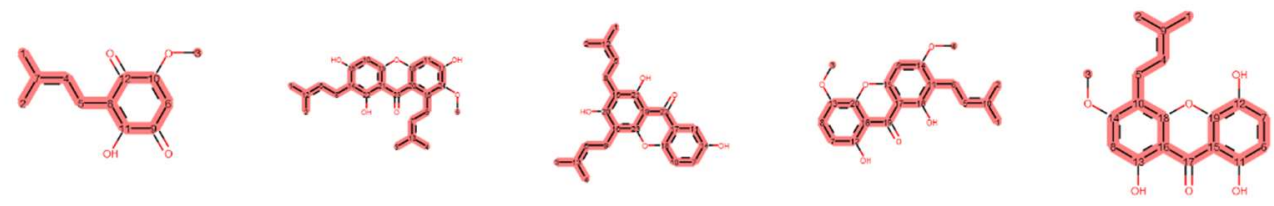

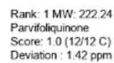

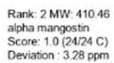
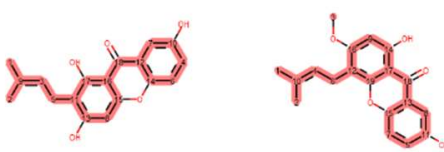

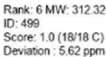

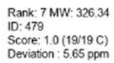

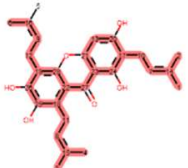

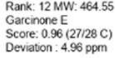

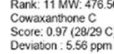

Sont
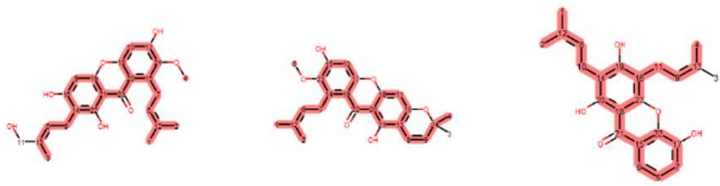

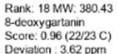

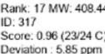

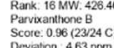

Deviaton. $5.82 \mathrm{ppm}$
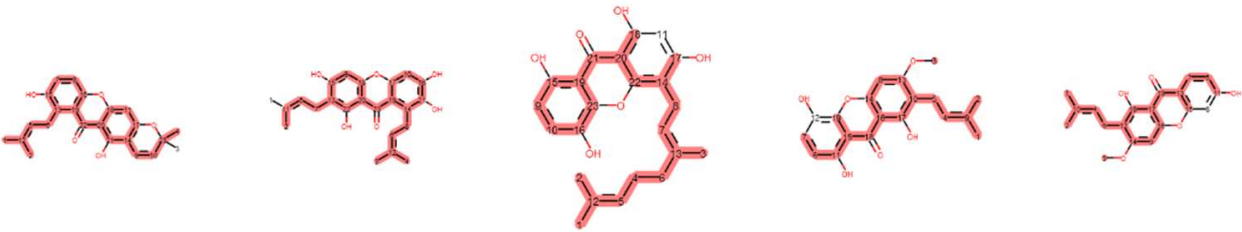

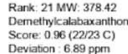

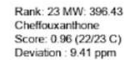

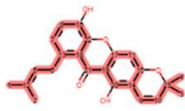

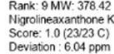

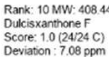

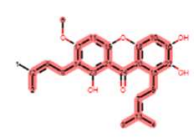

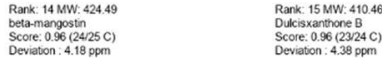

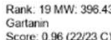
Deviaton $4.85 \mathrm{pph}$

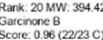

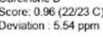

Figure S27. Part of displayed results for the ${ }^{13} \mathrm{C}-\mathrm{NMR}$ dereplication (+ DEPT 90 and 135 ) (175 MHz) of the mangosteen (fruit peel) fraction based on Garcinia DB. Equivalent carbons were allowed. 


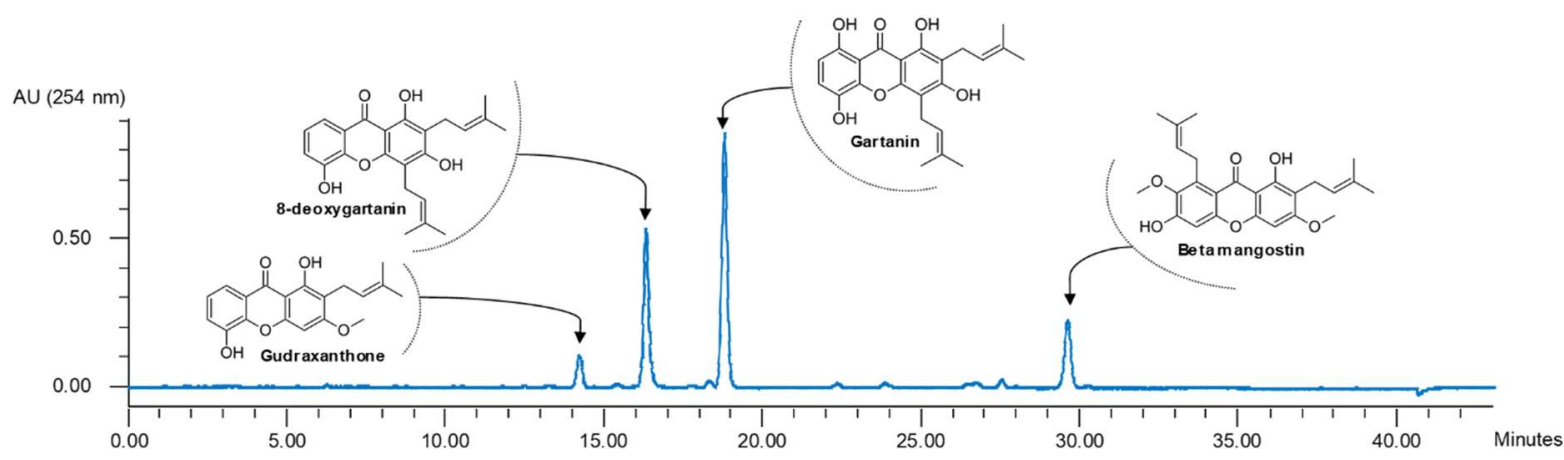

Figure S28. HPLC-UV ( $\lambda 254 \mathrm{~nm}$ ) chromatogram of the fraction from the Garcina mangostana (fruit peel) cyclohexanic extract containing minor xanthones.
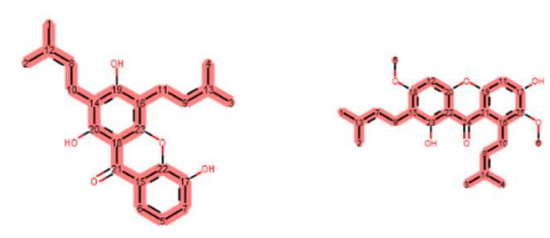

Rank: $1(23223 \mathrm{C}$
$8-\mathrm{deoxygartanin}$

8-deoxygartanin
MW. 380.43
Score match: 1.0 Emor : 2.62
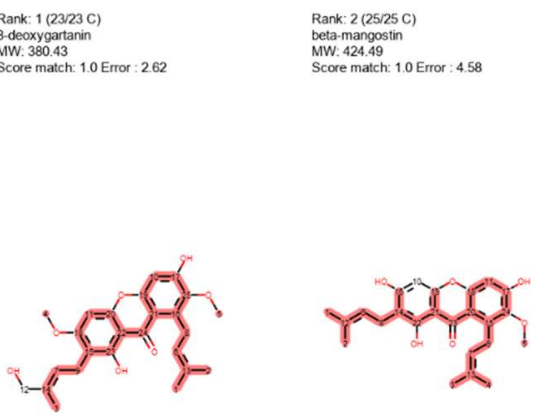
Rank: $3(1919 \mathrm{C})$
ID: 447
ID: 447
MW. 342.34
Score match: 1.0 Emor : 5.33

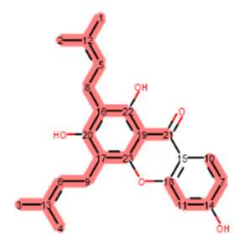

Rank: $8(22 / 23 \mathrm{C}$
Garcinone A

MW: 380.43

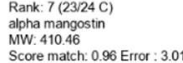

Rank: 6 (24/25 C)
Fuscaxanthone D

MW: 400.49
Score match: 0.96 Error 489

Score match: 0.96 Error : 3.0
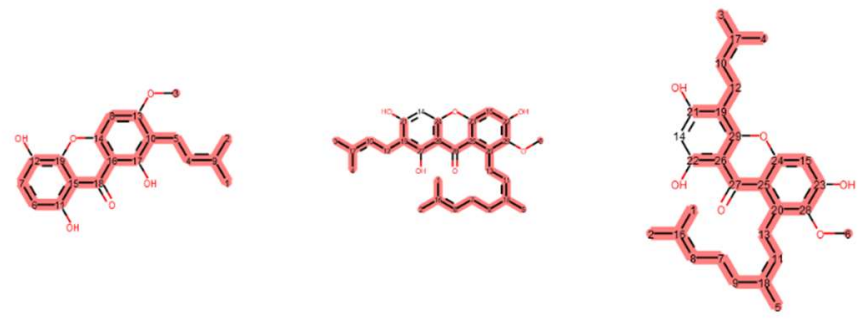

Rank: 4 (28/29 C)
Cowanin
MW: 478.58
Score match: 0.97 Error : 7.49

Rank: $5(28299 \mathrm{C})$

Isocowanin
MW: 478.58
Score match 0.97 Error : 10.08
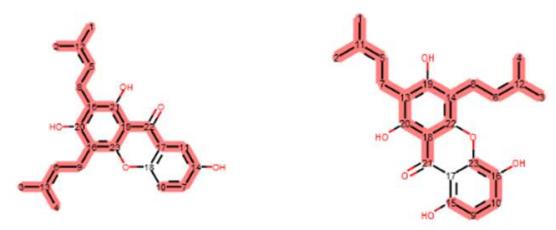

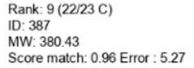

Rank: $10(222323$

MW: 396.43

Figure S29. Part of displayed results for the ${ }^{13}$ C-NMR dereplication (+ DEPT 90 and 135) of the mangosteen (fruit peel) fraction based on Garcinia DB. Equivalent carbons were allowed. 


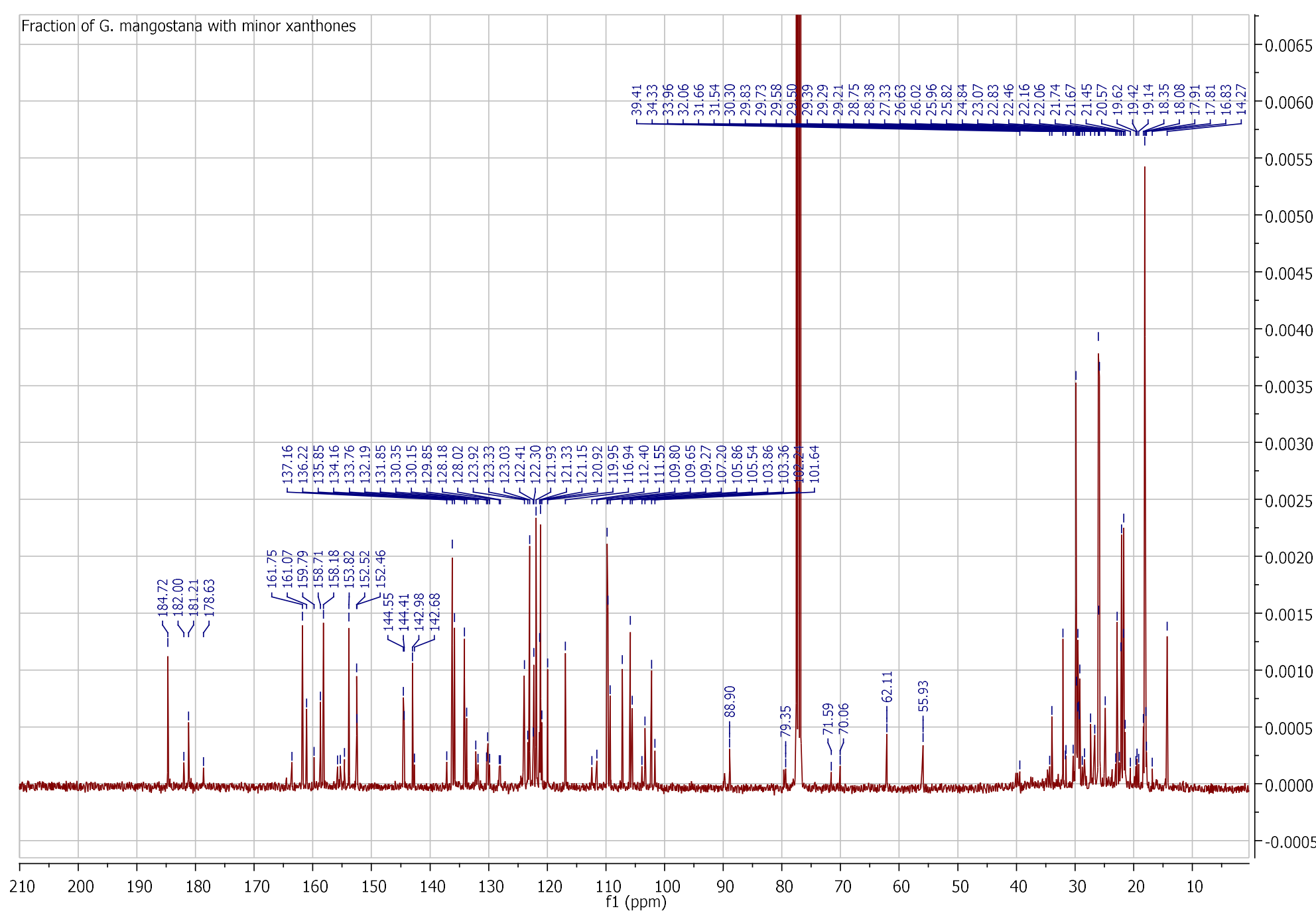

Figure S30. ${ }^{13} \mathrm{C}-\mathrm{NMR}$ spectrum ( 12000 scans) of the fraction from mangosteen (fruit peel) cyclohexanic extract ( $30 \mathrm{mg}$ ) containing minor xanthones recorded in $\mathrm{CDCl}{ }_{3}$ at $100 \mathrm{MHz}$ 


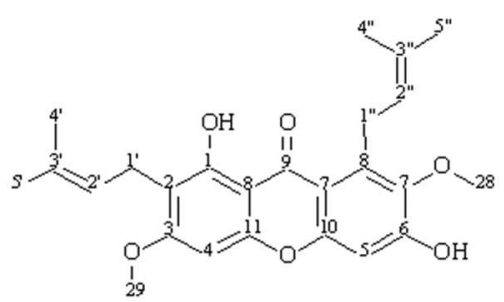

Rank: 1 (25/25 C)

Beta-mangostin

MW: 424.49 Score: 1.0

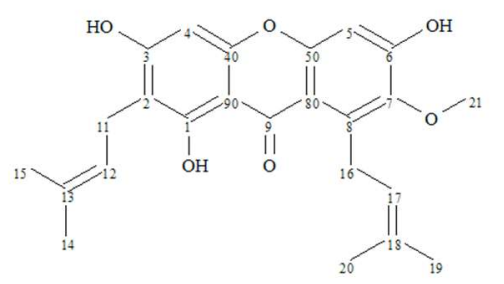

Rank: 4 (23/24 C)

alpha-mangostin

MW: 410.5 Score: 0.96

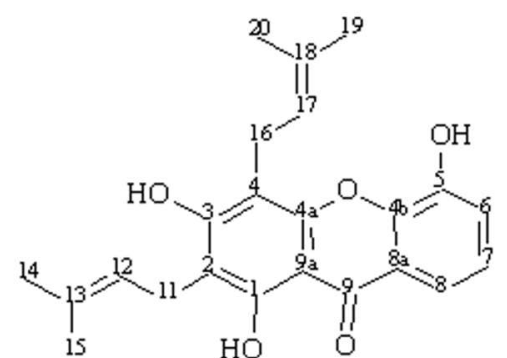

Rank: 2 (23/23 C)

8-Desoxygartanin

MW: 380.44 Score: 1.0

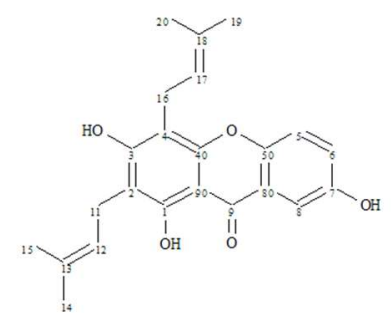

Rank: $5(22 / 23 \mathrm{C})$

52601-11-3

MW: 380.4 Score: 0.96

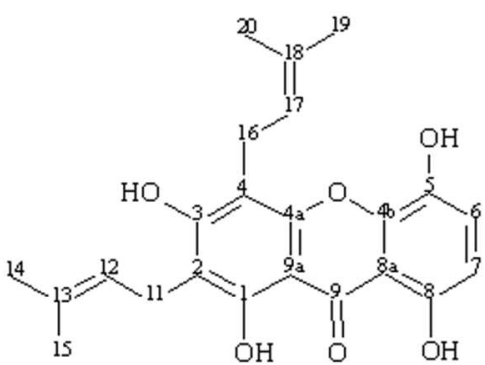

Rank: 3 (23/23 C)

Gartanin

MW: 396.44 Score: 1.0

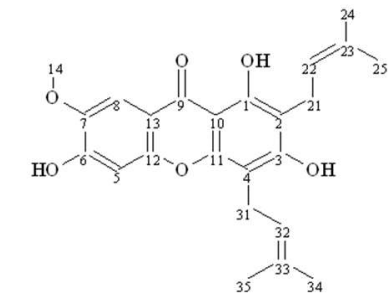

Rank: 6 (23/24 C)

cochinchinone $\mathrm{E}$

MW: 410.5 Score: 0.96

Figure S31. Part of displayed results for the ${ }^{13}$ C-NMR dereplication (+ DEPT 90 and 135) of the mangosteen (fruit peel) fraction based on CH-NMR-NP DB. Equivalent carbons were allowed. 
Table S1. (-)-noscapine $\delta_{\mathrm{C}}$ in the poppy extract and predicted (ACD/Labs NMR Predictor) values. $\Delta \delta_{\mathrm{C}}>1.3$ ppm are highlighted in yellow.

\begin{tabular}{|c|c|c|}
\hline \multicolumn{3}{|c|}{$(-)$-noscapine } \\
\hline $\begin{array}{l}\text { Observed } \delta_{\mathrm{C}}(\mathrm{ppm}) \text { in poppy extract } \\
\qquad\left(\mathrm{CDCl}_{3}\right)\end{array}$ & Predicted $\delta_{\mathrm{C}}(\mathbf{p p m})$ & $\Delta \delta_{\mathrm{c}}(\mathrm{ppm})$ \\
\hline 168.3 & 166.9 & 1.4 \\
\hline 152.3 & 152.6 & 0.3 \\
\hline 148.0 & 148.5 & 0.0 \\
\hline 147.8 & 147.8 & 0.0 \\
\hline 141.2 & 145.8 & 4.6 \\
\hline 140.7 & 140.5 & 0.3 \\
\hline 134.1 & 134.1 & 0.0 \\
\hline 132.2 & 129.7 & 2.5 \\
\hline 120.3 & 120.6 & 0.3 \\
\hline 119.0 & 119.5 & 0.5 \\
\hline 118.2 & 118.6 & 0.4 \\
\hline 117.9 & 117.2 & 0.7 \\
\hline 102.5 & 102.4 & 0.0 \\
\hline 100.9 & 100.8 & 0.1 \\
\hline 82.0 & 82.3 & 0.3 \\
\hline 62.5 & 62.0 & 0.5 \\
\hline 61.0 & 63.1 & 2.1 \\
\hline 59.5 & 59.4 & 0.1 \\
\hline 56.9 & 56.7 & 0.2 \\
\hline 50.0 & 49.1 & 0.9 \\
\hline 46.3 & 44.3 & 2.0 \\
\hline 22.8 & 23.5 & 0.7 \\
\hline
\end{tabular}


Table S2. ${ }^{13} \mathrm{C}$ NMR spectrum of papaverine recorded in $\mathrm{CDCl}_{3}$ at $100 \mathrm{MHz}$

\begin{tabular}{|c|c|}
\hline \multicolumn{2}{|c|}{ Papaverine } \\
\hline Carbon number (type) & $\delta_{13 C}(p p m)$ \\
\hline $1(\mathrm{Cq})$ & 157.8 \\
\hline $3(\mathrm{CH})$ & 141.0 \\
\hline $4(\mathrm{CH})$ & 118.8 \\
\hline $4 a(C q)$ & 133.5 \\
\hline $5(\mathrm{CH})$ & 105.3 \\
\hline $6(\mathrm{Cq})$ & 152.4 \\
\hline $7(\mathrm{Cq})$ & 149.8 \\
\hline $8(\mathrm{CH})$ & 104.2 \\
\hline $8 \mathrm{a}(\mathrm{Cq})$ & 123.0 \\
\hline $1^{\prime}(\mathrm{Cq})$ & 132.2 \\
\hline $2^{\prime}(\mathrm{CH})$ & 111.8 \\
\hline $3^{\prime}(\mathrm{Cq})$ & 149.0 \\
\hline $4^{\prime}(\mathrm{Cq})$ & 147.5 \\
\hline $5^{\prime}(\mathrm{CH})$ & 111.1 \\
\hline $6^{\prime}(\mathrm{CH})$ & 120.5 \\
\hline $\mathrm{OMe}$ & 56.1 \\
\hline $\mathrm{OMe}(\mathrm{X} 2)$ & 55.9 \\
\hline $\mathrm{OMe}$ & 55.8 \\
\hline $\mathrm{CH}_{2}$ & 42.3 \\
\hline
\end{tabular}<smiles>COc1ccc(Cc2nccc3cc(OC)c(OC)cc23)cc1OC</smiles> 
Table S3. Retention time $\left(R_{t}\right)$ and ESI-MS ${ }^{2}$ data for the major compounds identified in the rosemary leaf MeOH extract

\begin{tabular}{|c|c|c|c|c|c|c|c|c|}
\hline Peak & $\begin{array}{c}t_{R} \\
(\min )\end{array}$ & UV $\lambda_{\max }(\mathbf{n m})$ & $\begin{array}{c}(+)-E S I-M S \\
m / z\end{array}$ & $\begin{array}{c}(+)-E S I-M S^{2} \\
m / z\end{array}$ & $\begin{array}{c}\text { (-)-ESI-MS } \\
m / z\end{array}$ & $\begin{array}{c}\text { (-)-ESI-MS } \\
m / z\end{array}$ & $\begin{array}{c}\text { Suggested } \\
\text { molecular weight } \\
\text { (Da) }\end{array}$ & Hypothetical structure \\
\hline 1 & 3.0 & - & $\begin{array}{l}325\left[\mathbf{M}+\mathbf{H}-\mathrm{H}_{2} \mathbf{O}\right]^{+} \\
343[\mathrm{M}+\mathrm{H}]^{+} \\
360\left[\mathrm{M}+\mathrm{H}+\mathrm{H}_{2} \mathrm{O}\right]^{+}\end{array}$ & $\begin{array}{l}145\left[\mathrm{M}+\mathrm{H}-\mathrm{H}_{2} \mathrm{O}-\right. \\
180]^{+} / 127 / 163 / 97 / \\
264 / 292 / 307 / 247 / 109 / 2 \\
23 / 186 \\
\end{array}$ & $\begin{array}{l}\mathbf{6 8 3}[\mathbf{2 M}-\mathrm{M}]^{-} \\
341[\mathrm{M}-\mathrm{H}]^{-}\end{array}$ & $341[\mathrm{M}-\mathrm{H}]^{-}$ & 342 & Disaccharide (sucrose ?) \\
\hline 2 & 20.6 & 202,329 & $\begin{array}{l}743[2 \mathrm{M}+\mathrm{Na}]^{+} \\
383[\mathrm{M}+\mathrm{Na}]^{+} \\
\end{array}$ & $383[\mathrm{M}+\mathrm{Na}]^{+}$ & $\begin{array}{l}719\left[^{72 M-H}\right]^{-} \\
359[\mathrm{M}-\mathrm{H}]^{-}\end{array}$ & $359[\mathrm{M}-\mathrm{H}]^{-}$ & 360 & Rosmarinic acid \\
\hline
\end{tabular}

Table S4. Retention time $\left(R_{t}\right)$ and ESI-MS ${ }^{2}$ data for the major compounds identified in the rosemary leaf DCM and E392 extracts

\begin{tabular}{|c|c|c|c|c|c|c|c|c|}
\hline Peak & $\begin{array}{c}\text { tR } \\
(\mathrm{min})\end{array}$ & $\mathbf{U V} \lambda_{\max }(\mathbf{n m})$ & $\begin{array}{c}(+)-\text { ESI-MS } \\
m / z\end{array}$ & $\begin{array}{c}{ }^{(+)-E S I-M S}{ }^{2} \\
m / z\end{array}$ & $\begin{array}{c}(-)-E S I-M S \\
m / z\end{array}$ & $\begin{array}{c}\text { (-)-ESI-MS } \\
m / z\end{array}$ & $\begin{array}{c}\text { Suggested } \\
\text { molecular weight } \\
\text { (Da) }\end{array}$ & Hypothetical structure $^{1}$ \\
\hline 1 & 12.7 & 282 & $\begin{array}{l}331[\mathrm{M}+\mathrm{H}]^{+} \\
683[2 \mathrm{M}+\mathrm{Na}]^{+}\end{array}$ & $\begin{array}{l}285\left[\mathrm{M}-\mathrm{CO}_{2} \mathrm{H}\right]^{+} \\
267[\mathrm{M}+\mathrm{H}-64]^{+}\end{array}$ & $\begin{array}{l}329[\mathrm{M}-\mathrm{H}]^{-} \\
659[2 \mathrm{M}-\mathrm{H}]^{-}\end{array}$ & $285\left[\mathrm{M}-\mathrm{H}-\mathrm{CO}_{2}\right]^{-}$ & 330 & Carnosol \\
\hline 2 & 19.1 & 284 & $333[\mathrm{M}+\mathrm{H}]^{+}$ & $287\left[\mathrm{M}-\mathrm{CO}_{2} \mathrm{H}\right]^{+}$ & $\begin{array}{l}331[\mathrm{M}-\mathrm{H}]^{-} \\
685[2 \mathrm{M}-2 \mathrm{H}+\mathrm{Na}]^{-}\end{array}$ & $287\left[\mathrm{M}-\mathrm{H}-\mathrm{CO}_{2}\right]^{-}$ & 332 & Carnosic acid \\
\hline 3 & 31.9 & - & $\begin{array}{l}55[\mathrm{M}+\mathrm{H}]^{+} \\
437\left[\mathrm{M}-\mathrm{H}_{2} \mathrm{O}+\mathrm{H}\right]^{+}\end{array}$ & $\begin{array}{l}409\left[\mathrm{M}-\mathrm{CO}_{2} \mathrm{H}\right]^{+} \\
437\left[\mathrm{M}-\mathrm{H}_{2} \mathrm{O}+\mathrm{H}\right]^{+}\end{array}$ & $453[\mathrm{M}-\mathrm{H}]^{-}$ & $405[\mathrm{M}-\mathrm{H}-48]^{-}$ & 454 & Micromeric acid \\
\hline 4 & 36,7 & - & $\begin{array}{l}457[\mathrm{M}+\mathrm{H}]^{+} \\
439\left[\mathrm{M}-\mathrm{H}_{2} \mathrm{O}+\mathrm{H}\right]^{+}\end{array}$ & $393[\mathrm{M}+\mathrm{H}-64]^{+}$ & $455[\mathrm{M}-\mathrm{H}]^{-}$ & $455[\mathrm{M}-\mathrm{H}]^{-}$ & 456 & Betulinic acid \\
\hline 5 & 40,2 & - & $\begin{array}{l}457[\mathrm{M}+\mathrm{H}]^{+} \\
439\left[\mathrm{M}-\mathrm{H}_{2} \mathrm{O}+\mathrm{H}\right]^{+}\end{array}$ & $\begin{array}{l}411\left[\mathrm{M}-\mathrm{CO}_{2} \mathrm{H}\right]^{+} \\
439\left[\mathrm{M}-\mathrm{H}_{2} \mathrm{O}+\mathrm{H}\right]^{+}\end{array}$ & $455[\mathrm{M}-\mathrm{H}]^{-}$ & $\begin{array}{l}455[\mathrm{M}-\mathrm{H}]^{-} \\
407[\mathrm{M}-\mathrm{H}-48]^{-}\end{array}$ & 456 & $\begin{array}{l}\text { Ursolic acid } \\
\text { / Oleanolic acid }\end{array}$ \\
\hline
\end{tabular}

\footnotetext{
${ }^{1}$ Kontogianni, V. G.; Tomic, G.; Nikolic, I.; Nerantzaki, A. A.; Sayyad, N.; Stosic-Grujicic, S.; Stojanovic, I.; Gerothanassis, I. P.; Tzakos, A. G. Food Chem. 2013, 136, 120-129.
} 
Table S5. $\delta_{\mathrm{C}}$ matching with triterpenes and carnosic acid in the E392 extract along with their respective predicted values $\left(\mathrm{ACD} / \mathrm{Labs} \mathrm{NMR}\right.$ Predictor). ${ }^{1} \Delta \delta_{\mathrm{C}}>1.3 \mathrm{ppm}$ are highlighted in yellow. Hidden and missing chemical shifts are highlighted in green and blue respectively.

\begin{tabular}{|c|c|c|c|c|c|c|c|c|c|c|}
\hline \multirow[b]{2}{*}{$\begin{array}{l}\text { Carbons } \\
\text { numbering }\end{array}$} & \multicolumn{2}{|c|}{ Betulinic acid } & \multicolumn{2}{|c|}{ Ursolic acid } & \multicolumn{2}{|c|}{ Micromeric acid } & \multicolumn{2}{|c|}{ Oleanolic acid } & \multicolumn{2}{|c|}{ Carnosic acid } \\
\hline & $\begin{array}{c}\delta_{\mathrm{C}}(\mathrm{ppm}) \\
\text { matched in } \\
\text { extract } \\
(\mathrm{DMSO}-\mathrm{d6})\end{array}$ & $\begin{array}{l}\text { Predicted } \delta_{\mathrm{C}} \\
\quad(\mathbf{p p m})\end{array}$ & $\begin{array}{c}\delta_{\mathrm{c}}(\mathrm{ppm}) \\
\text { matched } \\
\text { in extract } \\
(\mathrm{DMSO}-\mathrm{d6})^{2}\end{array}$ & $\begin{array}{l}\text { Predicted } \delta_{\mathrm{C}} \\
\quad(\mathrm{ppm})\end{array}$ & $\begin{array}{c}\delta_{\mathrm{c}}(\mathrm{ppm}) \\
\text { matched in } \\
\text { extract } \\
(\mathrm{DMSO}-\mathrm{d6})^{3}\end{array}$ & $\begin{array}{l}\text { Predicted } \delta_{\mathrm{C}} \\
\quad(\mathbf{p p m})\end{array}$ & $\begin{array}{c}\delta_{\mathrm{C}}(\mathrm{ppm}) \\
\text { matched in } \\
\text { extract } \\
(\mathrm{DMSO}-\mathrm{d6})\end{array}$ & $\begin{array}{l}\text { Predicted } \\
\delta_{\mathrm{c}}(\mathbf{p p m})\end{array}$ & $\begin{array}{c}\delta_{\mathrm{C}}(\mathrm{ppm}) \\
\text { matched in } \\
\text { extract } \\
(\mathrm{DMSO}-\mathrm{d6})\end{array}$ & $\begin{array}{c}\text { Predicted } \\
\delta_{\mathrm{c}}(\mathbf{p p m})\end{array}$ \\
\hline 1 & 38.3 & 38.2 & 38.3 & 39.0 & 38.3 & 38.5 & 38.3 & 39.1 & 33.9 & 33.9 \\
\hline 2 & 27.0 & 26.5 & 27.6 & 28.0 & 27.6 & 27.4 & 27.6 & 27.9 & 19.8 & 19.3 \\
\hline 3 & 76.9 & 78.4 & 76.9 & 78.2 & 76.9 & 78.4 & 76.9 & 78.3 & 40.9 & 41.6 \\
\hline 4 & 38.7 & 38.1 & 38.4 & 39.2 & 38.4 & 38.9 & 38.4 & 39.3 & 33.6 & 34.5 \\
\hline 5 & 53.5 & 54.0 & 54.8 & 55.6 & 54.8 & 55.2 & 54.8 & 56.0 & 53.5 & 53.9 \\
\hline 6 & 19.8 & 19.7 & 19.8 & 19.4 & 18.3 & 18.5 & 19.8 & 19.5 & 20.5 & 20.4 \\
\hline 7 & 33.9 & 34.1 & 33.3 & 33.3 & 33.4 & 33.0 & 33.9 & 33.6 & 32.4 & 32.1 \\
\hline 8 & 41.3 & 41.1 & - & 39.8 & 41.3 & 40.2 & - & 39.8 & 129.0 & 129.2 \\
\hline 9 & 50.0 & 50.3 & 48.5 & 48.0 & 48.5 & 47.9 & 48.5 & 48.2 & - & 121.7 \\
\hline 10 & 36.6 & 37.0 & 36.6 & 37.2 & 36.6 & 37.4 & 36.6 & 37.5 & 46.8 & 47.3 \\
\hline 11 & 20.5 & 21.0 & 23.8 & 23.5 & 23.8 & 23.8 & 23.8 & 23.8 & - & 142.5 \\
\hline 12 & 25.1 & 25.9 & 125.0 & 125.4 & 124.6 & 124.6 & 121.6 & 121.7 & - & 141.3 \\
\hline 13 & 38.5 & 38.6 & 139.1 & 139.0 & 139.0 & 139.0 & 145.2 & 144.7 & 134.3 & 133.9 \\
\hline 14 & 42.4 & 42.8 & 42.4 & 42.4 & 42.4 & 42.3 & 42.4 & 42.6 & 121.6 & 119.1 \\
\hline 15 & 31.2 & 30.7 & 29.2 & 28.7 & 29.3 & 28.2 & 29.3 & 28.3 & 26.0 & 27.2 \\
\hline 16 & 32.4 & 32.7 & 25.1 & 24.8 & 25.1 & 24.2 & 27.0 & 27.0 & 22.7 & 22.4 \\
\hline 17 & 55.4 & 56.5 & 47.1 & 48.1 & - & 49.0 & 46.8 & 47.3 & 22.7 & 22.4 \\
\hline 18 & 48.5 & 48.7 & 53.5 & 53.4 & 52.4 & 52.8 & 40.8 & 41.7 & 32.9 & 2.8 \\
\hline 19 & 47.2 & 48.6 & 38.6 & 39.4 & 40.8 & 39.8 & - & 45.3 & 23.0 & 22.1 \\
\hline 20 & 150.3 & 151.0 & 38.5 & 39.2 & 153.0 & 151.9 & 31.4 & 31.0 & 177.7 & 180.6 \\
\hline
\end{tabular}

${ }^{2}$ Chemical shifts described in Brandão, G., Kroon, E., Souza, D., Filho, J., Oliveira, A., 2013. Chemistry and Antiviral Activity of Arrabidaea pulchra (Bignoniaceae). Molecules 18, 9919-9932. https://doi.org/10.3390/molecules 18089919

${ }^{3}$ Micromeric acid was purified and NMR experiments were registered in DMSO-d6. 
Bruguière et al. Supporting information

\begin{tabular}{|c|c|c|c|c|c|c|c|c|}
\hline 21 & 30.2 & 30.5 & 31.2 & 31.1 & 31.2 & 30.7 & - & 34.7 \\
\hline 22 & 36.4 & 37.5 & 36.4 & 37.3 & 36.4 & 37.2 & 33.9 & 33.6 \\
\hline 23 & 27.4 & 27.3 & 27.4 & 27.4 & 27.6 & 27.4 & 27.4 & 27.4 \\
\hline 24 & 28.9 & 29.3 & 28.9 & 29.4 & 28.9 & 29.4 & 28.3 & 29.4 \\
\hline 25 & 16.0 & 16.0 & 15.1 & 15.0 & 15.1 & 14.9 & 15.1 & 14.9 \\
\hline 26 & 16.1 & 16.2 & 17.1 & 17.2 & 16.9 & 17.0 & 17.1 & 17.3 \\
\hline 27 & 15.1 & 14.9 & 23.3 & 23.9 & 23.3 & 24.0 & 25.6 & 26.1 \\
\hline 28 & 178.6 & 179.5 & 178.4 & 180.3 & 177.3 & 181.0 & 178.6 & 179.7 \\
\hline 29 & 109.7 & 109.8 & 16.9 & 17.4 & 104.6 & 106.2 & - & 30.2 \\
\hline 30 & 19.0 & 19.5 & 21.1 & 21.4 & 17.1 & 17.1 & 28.9 & 29.3 \\
\hline
\end{tabular}


Table S6. ${ }^{13} \mathrm{C}$ NMR spectrum of carnosol recorded in DMSO-d6 at $100 \mathrm{MHz}$

\begin{tabular}{|c|c|}
\hline \multicolumn{2}{|c|}{ Carnosol } \\
\hline Carbon number (type) & $\delta_{13 \mathrm{C}}(\mathrm{ppm})$ \\
\hline $1\left(\mathrm{CH}_{2}\right)$ & 29.5 \\
\hline $2\left(\mathrm{CH}_{2}\right)$ & 18.8 \\
\hline $3\left(\mathrm{CH}_{2}\right)$ & 40.8 \\
\hline $4(\mathrm{Cq})$ & 34.4 \\
\hline $5(\mathrm{CH})$ & 45.2 \\
\hline $6\left(\mathrm{CH}_{2}\right)$ & 29.1 \\
\hline $7(\mathrm{CH})$ & 77.3 \\
\hline $8(\mathrm{Cq})$ & 131.9 \\
\hline $9(\mathrm{Cq})$ & 122.1 \\
\hline $10(\mathrm{Cq})$ & 48.1 \\
\hline $11(\mathrm{Cq})$ & 143.6 \\
\hline $12(\mathrm{Cq})$ & 143.3 \\
\hline $13(\mathrm{Cq})$ & 134.6 \\
\hline $14(\mathrm{CH})$ & 111.6 \\
\hline $15(\mathrm{CH})$ & 26.5 \\
\hline $16\left(\mathrm{CH}_{3}\right)$ & 22.9 \\
\hline $17\left(\mathrm{CH}_{3}\right)$ & 19.7 \\
\hline $18\left(\mathrm{CH}_{3}\right)$ & 23.1 \\
\hline $19\left(\mathrm{CH}_{3}\right)$ & 31.6 \\
\hline $20(\mathrm{Cq})$ & 176.1 \\
\hline
\end{tabular}

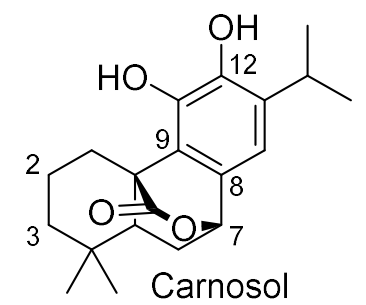

1 
Table S7. $8 \mathrm{c}(100 \mathrm{MHz})$ matching with xanthones in the mangosteen cyclohexanic extract along with their respective predicted values $(\mathrm{ACD} / \mathrm{Labs} \mathrm{NMR}$ Predictor). $\Delta \delta \mathrm{c}>1.3 \mathrm{ppm}$ are highlighted in yellow. Hidden and missing chemical shifts are highlighted in green and blue respectively.

\begin{tabular}{|c|c|c|c|c|c|c|c|c|c|c|}
\hline \multirow[b]{2}{*}{$\begin{array}{c}\text { Carbons } \\
\text { numbering }\end{array}$} & \multicolumn{2}{|c|}{$\alpha$-mangostin ${ }^{4}$} & \multicolumn{2}{|c|}{ Garcinone $\mathrm{E}^{5}$} & \multicolumn{2}{|c|}{$\gamma$-mangostin ${ }^{6}$} & \multicolumn{2}{|c|}{ Gartanin $^{7}$} & \multicolumn{2}{|c|}{ 8-deoxygartanin 7} \\
\hline & $\begin{array}{c}\boldsymbol{\delta}_{\mathrm{C}}(\mathbf{p p m}) \\
\text { matched in } \\
\text { extract } \\
\left(\mathrm{CDCl}_{3}\right)\end{array}$ & $\begin{array}{l}\text { Predicted } \delta_{c} \\
\quad(\mathbf{p p m})\end{array}$ & $\begin{array}{c}\boldsymbol{\delta}_{\mathrm{C}}(\mathbf{p p m}) \\
\text { matched in } \\
\text { extract } \\
\left(\mathrm{CDCl}_{3}\right)\end{array}$ & $\begin{array}{l}\text { Predicted } \delta_{c} \\
\quad(\mathbf{p p m})\end{array}$ & $\begin{array}{c}\delta_{\mathrm{C}}(\mathbf{p p m}) \\
\text { matched in } \\
\text { extract } \\
\left(\mathrm{CDCl}_{3}\right)\end{array}$ & $\begin{array}{l}\text { Predicted } \delta_{c} \\
\quad(\mathbf{p p m})\end{array}$ & $\begin{array}{c}\boldsymbol{\delta}_{\mathrm{C}}(\mathbf{p p m}) \\
\text { matched in } \\
\text { extract } \\
\left(\mathrm{CDCl}_{3}\right)\end{array}$ & $\begin{array}{l}\text { Predicted } \delta_{c} \\
\quad(\mathbf{p p m})\end{array}$ & $\begin{array}{c}\boldsymbol{\delta}_{\mathrm{C}}(\mathrm{ppm}) \\
\text { matched in } \\
\text { extract } \\
\left(\mathrm{CDCl}_{3}\right)\end{array}$ & $\begin{array}{l}\text { Predicted } \delta_{\mathrm{c}} \\
\quad(\mathbf{p p m})\end{array}$ \\
\hline 1 & 160.7 & 160.7 & 161.1 & 160.9 & 161.7 & 162.8 & 158.8 & 158.5 & 158.8 & 158.7 \\
\hline 2 & 108.4 & 108.4 & 109.3 & 109.3 & 108.6 & 108.6 & 111.4 & 110.6 & 109.3 & 109.1 \\
\hline 3 & 161.6 & 161.6 & 161.8 & 162.4 & 161.8 & 161.8 & 161.8 & 162.0 & 161.1 & 160.9 \\
\hline 4 & 93.4 & 93.3 & 93.4 & 93.3 & 93.4 & 93.0 & 107.3 & 106.8 & 109.3 & 109.1 \\
\hline $4 a$ & 155.2 & 155.1 & 155.9 & 156.8 & 155.9 & 155.8 & 152.6 & 153.1 & 152.6 & 152.4 \\
\hline 5 & 101.7 & 101.6 & 113.4 & 114.0 & 101.7 & 101.2 & 137.2 & 136.9 & 144.6 & 144.5 \\
\hline 6 & 154.7 & 154.5 & 148.9 & 148.7 & 148.9 & 149.6 & 123.3 & 123.9 & - & 119.7 \\
\hline 7 & 142.7 & 142.6 & - & 139.8 & 142.7 & 141.8 & - & 109.9 & 123.3 & 123.8 \\
\hline 8 & 137.2 & 137.1 & 128.4 & 128.0 & 132.3 & 131.4 & 153.9 & 154.1 & - & 116.9 \\
\hline $8 \mathrm{a}$ & 112.3 & 112.3 & 111.4 & 110.7 & 111.4 & 110.8 & 108.4 & 107.8 & 121.0 & 120.9 \\
\hline 9 & 182.2 & 182.1 & 183.2 & 183.2 & 183.2 & 183.3 & 184.8 & 185.4 & 181.3 & 181.1 \\
\hline $9 a$ & 103.7 & 103.7 & 103.5 & 103.0 & 103.7 & 103.8 & 102.4 & 102.5 & 103.5 & 103.3 \\
\hline $10 \mathrm{a}$ & 155.2 & 155.8 & 152.6 & 152.4 & 153.9 & 153.6 & 144.6 & 143.9 & 143.0 & 144.3 \\
\hline $1^{\prime}$ & 21.6 & 21.5 & 21.7 & 21.7 & 22.1 & 22.1 & 22.1 & 22.1 & 21.6 & 21.6 \\
\hline $2^{\prime}$ & 121.6 & 121.4 & 121.7 & 122.2 & 123.3 & 123.6 & 121.8 & 121.9 & 121.2 & 121.2 \\
\hline $3^{\prime}$ & 135.9 & 135.8 & 134.1 & 134.2 & 132.3 & 131.4 & 134.1 & 134.6 & 136.4 & 136.3 \\
\hline $4^{\prime}$ & 18.1 & 18.0 & 18.1 & 18.0 & 18.1 & 18.1 & 18.0 & 18.0 & 18.1 & 18.0 \\
\hline 5 & 25.8 & 25.8 & 26.0 & 25.9 & 26.0 & 26.0 & 26.0 & 25.9 & 26.0 & 25.9 \\
\hline 1" & 26.7 & 26.6 & 26.0 & 26.1 & 26.7 & 26.5 & 21.8 & 22.0 & 22.1 & 22.1 \\
\hline
\end{tabular}

${ }^{4}$ Experimental $\delta_{\mathrm{C}}$ in $\mathrm{CDCl}_{3}$ can be found in Han, A.-R.; Kim, J.-A.; Lantvit, D. D.; Kardono, L. B. S.; Riswan, S.; Chai, H.; Carcache de Blanco, E. J.; Farnsworth, N. R.; Swanson, S. M.; Kinghorn, A. D., Cytotoxic xanthone constituents of the stem bark of Garcinia mangostana (Mangosteen). J. Nat. Prod. 2009, 72 (11), 2028-2031.

${ }^{5}$ Experimental $\delta_{\mathrm{C}}$ in $\mathrm{CDCl}_{3}$ can be found in Sakai, S.-I.; Katsura, M.; Takayama, H.; Aimi, N.; Chokethaworn, N.; Suttajit, M., The structure of garcinone E. Chem. Pharm. Bull. 1993, 41 (5), 958-960.

${ }^{6}$ To our knowledge, no experimental $\delta_{\mathrm{C}}$ were described in $\mathrm{CDCl}_{3}$

${ }^{7}$ Experimental $\delta_{\mathrm{C}}$ in $\mathrm{CDCl}_{3}$ can be found in Groweiss, A.; Cardellina, J. H.; Boyd, M. R., HIV-Inhibitory prenylated xanthones and flavones from Maclura tinctoria. J. Nat. Prod. 2000, 63 (11), 1537-9. 
Bruguière et al. Supporting information

\begin{tabular}{|c|c|c|c|c|c|c|c|c|c|c|}
\hline $2 "$ & 123.3 & 123.2 & 121.7 & 122.4 & - & 124.6 & 123.3 & 123.9 & 121.8 & 122.2 \\
\hline 3" & 132.3 & 132.1 & 132.3 & 132.3 & 129.2 & 129.1 & 133.7 & 133.4 & 133.8 & 133.5 \\
\hline 4" & 18.2 & 18.2 & 18.1 & 18.1 & 18.1 & 18.1 & 18.2 & 18.1 & 18.1 & 18.0 \\
\hline $5 "$ & 25.8 & 25.8 & 26.0 & 25.9 & 25.8 & 25.9 & 25.8 & 25.8 & 25.8 & 25.7 \\
\hline 1’” & & & 22.7 & 22.7 & & & & & & \\
\hline 2"' & & & 121.2 & 120.8 & & & & & & \\
\hline 3"” & & & 133.8 & 132.9 & & & & & & \\
\hline 4"” & & & 18.2 & 18.0 & & & & & & \\
\hline $5^{\prime \prime \prime}$ & & & 25.8 & 25.8 & & & & & & \\
\hline $\mathrm{OMe}$ & 62.2 & 62.1 & & & & & & & & \\
\hline
\end{tabular}


Table S8. $\delta_{\mathrm{c}}(175 \mathrm{MHz})$ matching with xanthones in the mangosteen cyclohexanic extract along with their respective predicted values $\left(\mathrm{ACD} / \mathrm{Labs} \mathrm{NMR}\right.$ Predictor). $\Delta \delta_{\mathrm{C}}>1.3 \mathrm{ppm}$ are highlighted in yellow. Hidden and missing chemical shifts are highlighted in green and blue respectively. Methyls incorrectly considered as $\mathrm{CH}$ by MixONat due to their presence in dept-90.csv file are highlighted in orange.

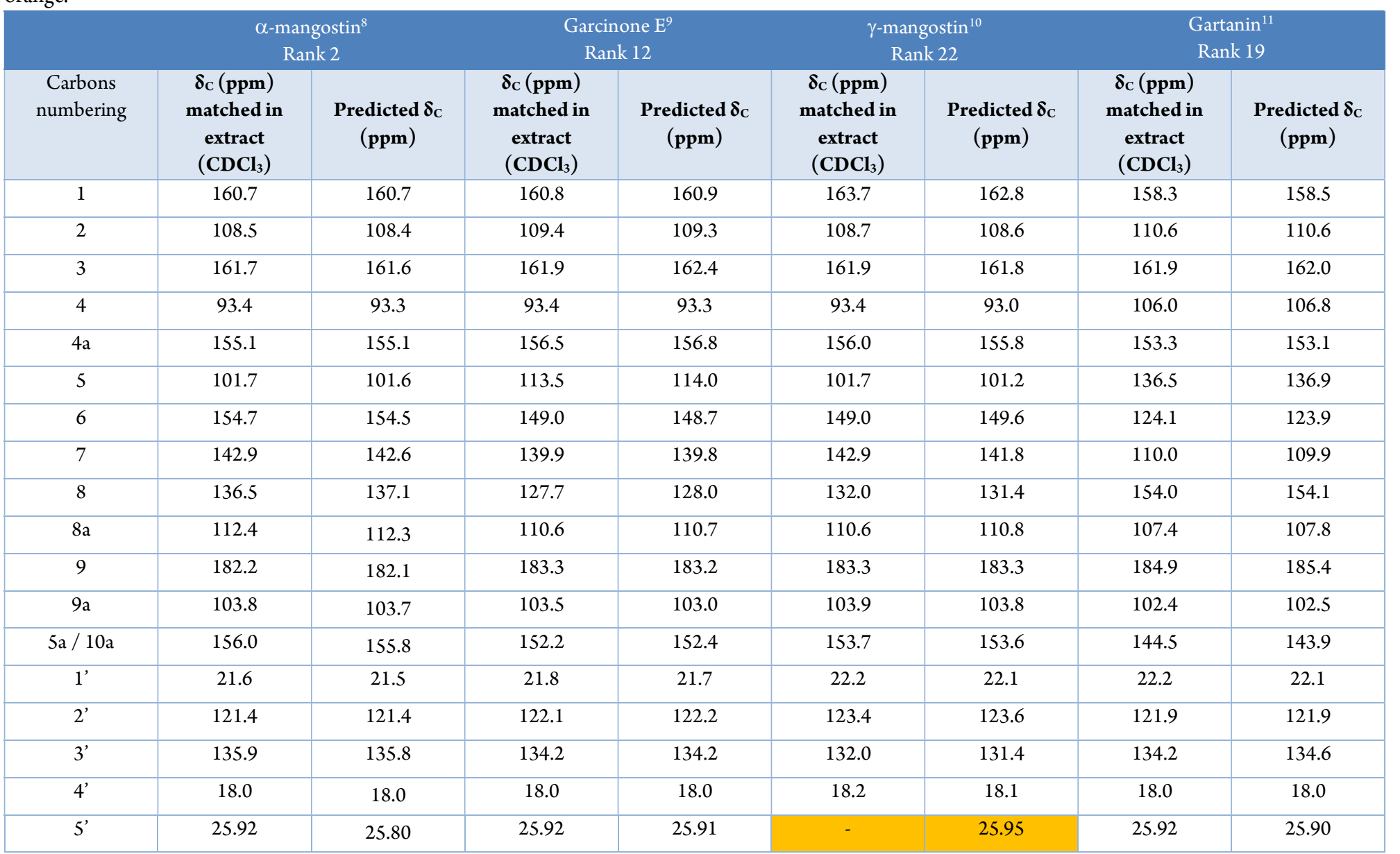

\footnotetext{
${ }^{8}$ Experimental $\delta_{\mathrm{C}}$ in $\mathrm{CDCl}_{3}$ can be found in Han, A.-R.; Kim, J.-A.; Lantvit, D. D.; Kardono, L. B. S.; Riswan, S.; Chai, H.; Carcache de Blanco, E. J.; Farnsworth, N. R.; Swanson, S. M.; Kinghorn, A. D., Cytotoxic xanthone constituents of the stem bark of Garcinia mangostana (Mangosteen). J. Nat. Prod. 2009, 72 (11), 2028-2031.

${ }^{9}$ Experimental $\delta_{\mathrm{C}}$ in $\mathrm{CDCl}_{3}$ can be found in Sakai, S.-I.; Katsura, M.; Takayama, H.; Aimi, N.; Chokethaworn, N.; Suttajit, M., The structure of garcinone E. Chem. Pharm. Bull. 1993, 41 (5), 958-960.

${ }^{10}$ To our knowledge, no experimental $\delta_{\mathrm{C}}$ were described in $\mathrm{CDCl}_{3}$
}

${ }^{11}$ Experimental $\delta_{\mathrm{C}}$ in $\mathrm{CDCl}_{3}$ can be found in Groweiss, A.; Cardellina, J. H.; Boyd, M. R., HIV-Inhibitory prenylated xanthones and flavones from Maclura tinctoria. J. Nat. Prod. 2000, 63 (11), 1537-9. 
Bruguière et al. Supporting information

\begin{tabular}{|c|c|c|c|c|c|c|c|c|}
\hline $1 "$ & 26.8 & 26.6 & 26.2 & 26.1 & 26.8 & 26.5 & 21.9 & 22.0 \\
\hline 2" & 123.1 & 123.2 & 122.4 & 122.4 & 124.1 & 124.6 & 124.4 & 122.4 \\
\hline 3" & 132.2 & 132.1 & 132.2 & 132.3 & 128.5 & 129.1 & 133.8 & 133.4 \\
\hline 4" & 18.3 & 18.2 & 18.2 & 18.1 & 18.2 & 18.1 & 18.2 & 18.1 \\
\hline $5^{\prime \prime}$ & 25.92 & 25.80 & 25.92 & 25.91 & 25.92 & 25.85 & - & 25.80 \\
\hline 1"” & & & 22.8 & 22.7 & & & & \\
\hline 2"” & & & 121.2 & 120.8 & & & & \\
\hline 3"' & & & 132.4 & 132.9 & & & & \\
\hline 4"' & & & 18.2 & 18.0 & & & & \\
\hline 5"' & & & & 25.80 & & & & \\
\hline OMe & 62.3 & 62.1 & & & & & & \\
\hline
\end{tabular}




\begin{tabular}{|c|c|c|c|c|c|c|}
\hline \multirow[b]{2}{*}{$\begin{array}{c}\text { Carbons } \\
\text { numbering }\end{array}$} & \multicolumn{2}{|c|}{$\begin{array}{c}\text { 8-deoxygartanin }{ }^{11} \\
\text { Rank } 18\end{array}$} & \multicolumn{2}{|c|}{$\begin{array}{c}\beta \text {-mangostin } \\
\text { Rank } 14\end{array}$} & \multicolumn{2}{|c|}{$\begin{array}{l}\text { 9-hydroxycalabaxanthone }{ }^{13} \\
\text { Rank } 17\end{array}$} \\
\hline & $\begin{array}{c}\delta_{\mathrm{c}}(\mathrm{ppm}) \\
\text { matched in } \\
\text { extract } \\
\left(\mathrm{CDCl}_{3}\right)\end{array}$ & $\begin{array}{l}\text { Predicted } \delta_{\mathrm{c}} \\
\quad(\mathrm{ppm})\end{array}$ & $\begin{array}{c}\delta_{\mathrm{c}}(\mathrm{ppm}) \\
\text { matched in } \\
\text { extract } \\
\left(\mathrm{CDCl}_{3}\right)\end{array}$ & $\begin{array}{l}\text { Predicted } \delta_{\mathrm{c}} \\
\quad(\mathrm{ppm})\end{array}$ & $\begin{array}{c}\delta_{\mathrm{C}}(\mathrm{ppm}) \\
\text { matched in } \\
\text { extract } \\
\left(\mathrm{CDCl}_{3}\right)\end{array}$ & $\begin{array}{l}\text { Predicted } \delta \mathrm{c} \\
\quad(\mathrm{ppm})\end{array}$ \\
\hline 1 & 158.9 & 158.7 & 159.4 & 159.4 & 158.1 & 157.9 \\
\hline 2 & 109.4 & 109.1 & 111.5 & 111.4 & 104.6 & 104.6 \\
\hline 3 & 160.8 & 160.9 & 163.7 & 163.5 & 160.7 & 160.6 \\
\hline 4 & 105.7 & 105.4 & 89.1 & 88.8 & 94.4 & 94.9 \\
\hline $4 a$ & 152.7 & 152.4 & 155.5 & 155.3 & 158.3 & 157.4 \\
\hline 5 & 144.5 & 144.3 & 101.7 & 101.5 & 101.7 & 101.5 \\
\hline 6 & 120.1 & 119.7 & 153.7 & 153.6 & 154.0 & 154.1 \\
\hline 7 & 124.1 & 123.8 & 142.9 & 142.7 & 142.9 & 142.8 \\
\hline 8 & 117.1 & 116.9 & 136.5 & 137.2 & 136.5 & 136.8 \\
\hline $8 a$ & 121.1 & 120.9 & 112.4 & 112.2 & 112.4 & 112.2 \\
\hline 9 & 180.9 & 181.1 & 182.8 & 182.7 & 181.4 & 181.7 \\
\hline $9 a$ & 103.5 & 103.3 & 101.4 & 101.1 & 103.8 & 103.8 \\
\hline $5 a / 10 a$ & 144.5 & 144.5 & 155.3 & 155.2 & 154.8 & 154.8 \\
\hline 10 & & & & & 115.9 & 115.6 \\
\hline 11 & & & & & 127.4 & 127.2 \\
\hline 12 & & & & & 78.2 & 77.3 \\
\hline 13 & & & & & - & 27.40 \\
\hline 14 & & & & & 28.5 & 29.4 \\
\hline $1^{\prime}$ & 21.7 & 21.6 & 21.3 & 21.4 & 26.8 & 26.6 \\
\hline $2^{\prime}$ & 121.2 & 121.2 & 122.4 & 122.4 & 123.3 & 123.4 \\
\hline $3^{\prime}$ & 136.5 & 136.3 & 132.0 & 132.0 & 132.0 & 131.9 \\
\hline $4^{\prime}$ & 18.0 & 18.0 & 18.2 & 17.8 & 18.2 & 18.2 \\
\hline
\end{tabular}

${ }^{12}$ Experimental $\delta_{\mathrm{C}}$ in $\mathrm{CDCl}_{3}$ can be found in Likhitwitayawuid, K.; Phadungcharoen, T.; Krungkrai, J., Antimalarial xanthones from Garcinia cowa. Planta Med. 1998, 64 (01), 70-72. ${ }^{13}$ Ryu, H. W.; Curtis-Long, M. J.; Jung, S.; Jin, Y. M.; Cho, J. K.; Ryu, Y. B.; Lee, W. S.; Park, K. H., Xanthones with neuraminidase inhibitory activity from the seedcases of Garcinia mangostana. Bioorg. Med. Chem. 2010, 18 (17), 6258-6264. 
Bruguière et al. Supporting information

\begin{tabular}{|c|c|c|c|c|c|c|}
\hline 5 & 25.92 & 25.90 & - & 25.81 & 25.92 & 25.90 \\
\hline $1 "$ & 22.2 & 22.1 & 26.8 & 26.6 & & \\
\hline $2 "$ & 122.1 & 122.2 & 123.1 & 123.0 & & \\
\hline 3" & 133.8 & 133.5 & 132.2 & 132.2 & & \\
\hline $4 "$ & 18.0 & 18.0 & 18.0 & 18.0 & & \\
\hline $5 "$ & - & 25.70 & 25.9 & 25.8 & & \\
\hline $1 " ”$ & & & & & & \\
\hline 2"' & & & & & & \\
\hline 3"” & & & & & & \\
\hline $4 " ”$ & & & & & & \\
\hline 5"' & & & & & & \\
\hline 3-OMe & & & 56.1 & 55.8 & & \\
\hline 7-OMe & & & 62.3 & 62.1 & 62.3 & 62.0 \\
\hline
\end{tabular}




\section{Detailed MixONat software.}

MixONat software is freely distributed at https://sourceforge.net/projects/mixonat

Inputs and parameters. A graphical user interface (GUI) was designed with Kivy, an open Python library source compatible with Linux, Windows and OS X.

The home tab of the MixONat software (Figure S2) allows the selection of the input files by the user, using a file browsing function. The input files must include at least a DB (.sdf), sorted by the C-typeGen program, and ${ }^{13} \mathrm{C}-\mathrm{NMR}$ data, imported as a table (.csv) of $\delta_{\mathrm{C}}$ values and intensities. The users are also encouraged to provide DEPT-135 and 90 data (.csv).

The second tab (Figure S3) displays all the different parameters that can be adjusted:

- The tolerance $(\varepsilon)$ reflects the accuracy of the used database, as the program compares each chemical shift in the experimental spectrum $\left(\delta_{13 \mathrm{C}}\right)$ with each chemical shift in the SDF $\left(\delta_{\mathrm{SDF}}\right)$ for each molecule. It considers that $\delta_{13 \mathrm{C}}$ matches with $\delta_{\mathrm{SDF}}$ if $\delta_{\mathrm{SDF}}-\varepsilon<\delta_{13 \mathrm{C}}<\delta_{\mathrm{SDF}}+\varepsilon$. The default value for $\varepsilon$ is $1.3 \mathrm{ppm}^{14,15}$

- The tolerance incrementation can be turned ON or OFF. If ON, the program will start the matching process with $\varepsilon=0.0$ and will then increment this value by steps of $0.1 \mathrm{ppm}$, until it reaches the $\varepsilon$ value chosen by the user. This will match first the chemical shifts which are closest together. If this parameter is OFF, the algorithm will match a $\delta_{\mathrm{SDF}}$ with the first $\delta_{13 \mathrm{C}}$ falling into the $\pm \varepsilon$ interval. It is recommended to leave this parameter ON, especially when using experimental DBs.

- The DEPT alignment parameter is necessary to associate a $\delta_{13 \mathrm{C}}$ to its corresponding carbon in the DEPT-135 and and 90 spectra. Since the chemical shifts are never exactly the same from a spectrum to another, the user has to indicate the accuracy of the alignment in each one of his spectra, the default value is $0.02 \mathrm{ppm}$. The quality of the results will be very dependent to the quality of this alignment.

- The equivalent carbon factor can be turn ON or OFF. Turning it ON will allow the same $\delta_{13 \mathrm{C}}$ to be matched multiple times if several identical $\delta_{\mathrm{SDF}}$ are found (equivalent carbons in the database). If OFF, a $\delta_{13 \mathrm{C}}$ can only be matched once, even if equivalent carbons are found in the database.

- The molecular weight filter will only show results if they correspond to the ones required by the user. One can either select specific mass, or a range of values. The algorithm will search for molecules with the indicated molecular weight(s).

- The number of results to display can be selected by the user, as well as the number of results per page when saving the files, making it easier to browse and analyze them.

- For each compound of the DB, a score is attributed and corresponds to the number of $\delta_{13 \mathrm{C}}$ matched with $\delta_{\mathrm{SDF}}$ out of the number of carbons of the compound. A minimal score to reach can also be a parameter to limit the number of results. If a molecule does not reach the minimal score, it will not be displayed; the minimum being 0.0 and the maximum 1.0. By default, the minimal score is 0.0 , in order to be able to see every result that fits the previous parameters.

- The directory of the output files can be selected by the user, using a browser function.

The third and last tab is the C-typeGen program that allows the user to create DBs compatible with the MixONat dereplication process. It sorts $\delta_{\mathrm{SDF}}$ by carbon types. Via a browser, the user must select the input files required, meaning the original database and the same database without stereochemistry. It is important that the stereochemistry is removed before the sorting, because it can alter the way the program works, resulting in a wrong carbon type listing. Even if this is a mandatory step before proceeding to the dereplication, this program is only used when creating a new database and is otherwise not required for the search itself.

Matching process. First, the program will start by sorting each carbon of the ${ }^{13} \mathrm{C}$ NMR spectrum depending on their type. This sorting is different, depending on the DEPT files provided by the user and according to the chosen DEPT alignment. If there is no DEPT data, then the carbon types are not differentiated. If a DEPT 135 has been given, carbons that have a negative intensity will be considered as methylenes, carbons with a positive intensity will be considered as methines or methyles. The rest of the chemical shifts from the ${ }^{13} \mathrm{C}-\mathrm{NMR}$ spectrum that are not visible in the DEPT-135 will be labeled as quaternaries. If DEPT-90 information is also provided, then the program will first consider that signals in the DEPT-90 are methines, before considering negative signals on the DEPT 135 as secondaries, positive signals on the DEPT 135 as primaries, and remaining carbons from the ${ }^{13} \mathrm{C}$ spectrum as quaternaries.

\footnotetext{
${ }^{14}$ The tolerance can be lowered when working with experimental DBs or increased if the DB was created with a less accurate prediction software.

${ }^{15}$ Bruguière, A.; Derbré, S.; Coste, C.; Le Bot, M.; Siegler, B.; Leong, S. T.; Sulaiman, S. N.; Awang, K.; Richomme, P. Fitoterapia 2018, 131, 59-64.
} 
In a second time, the matching process consists, for each compound of the $\mathrm{DB}$, in the comparison of $\delta_{13 \mathrm{C}}$ with $\delta_{\mathrm{SDF}}$. It is done by list of carbon types, meaning that the carbons labeled as quaternaries from the user spectra can only be matched with quaternary carbons from each compound of the SDF. All $\delta$ are first sorted by descending numerical order before the matching process. The program starts by the first molecule of the SDF, with the first $\delta_{\mathrm{SDF}}$, and tries to find a $\delta_{13 \mathrm{C}}$ that fits in the tolerance interval. As a reminder, if incrementation is $\mathrm{ON}$, the program will first look for perfect matches with $\varepsilon=0.0$, and gradually go up each cycle until $\varepsilon$ is the tolerance value chosen by the user. During the latter, the algorithm allows or not multiple uses of $\delta_{13 \mathrm{C}}$, depending on the equivalent carbon parameters. When all the $\delta_{\mathrm{SDF}}$ have been considered, the score and error of the molecule are calculated and stored. The score is defined as the number of $\delta_{13 \mathrm{C}}$ matched with $\delta_{\mathrm{SDF}}$ out of the number of carbons of the compound. a score of 1.0 thus means that all the carbons from the molecule have been matched; 0.50 means that only half of the signals were found. The error is the cumulated absolute difference between matched signals (i.e. $\left.\Sigma\left|\delta_{\mathrm{SDF}}-\delta_{13 \mathrm{C}}\right|\right)$. The algorithm will repeat the matching process for each molecule in the SDF which has a molecular weight fitting the parameters chosen by the user

In some rare cases, the algorithm matches couples of $\delta_{\mathrm{SDF}}$ and $\delta_{13 \mathrm{C}}$ values (within the same $\varepsilon$ window) with a non-optimal solution, increasing the error value. For example, the matching process associated $\delta_{\mathrm{SDF} 1}$ with $\delta_{13 \mathrm{C} 1}$ and $\delta_{\mathrm{SDF} 2}$ with $\delta_{13 \mathrm{C} 2}$ but, under closer inspection, if $\delta_{\mathrm{SDF} 1}$ was matched with $\delta_{13 \mathrm{C} 2}$ and $\delta_{\mathrm{SDF} 2}$ with $\delta_{13 \mathrm{C} 1}$, the total error on the molecule would be lower, and thus the molecule can see its rank increased. Thus, the very end of the process, another algorithm will optimize the local error on matched values.

Interactive results. At the end of the matching process, the number of desired molecules is displayed if they reach the minimal score fixed by the user. Compounds are ranked by decreasing score and increasing error, their structure, name, molecular weight, score and error (Figure S4). On the structure, matched carbons are highlighted in red. For each molecule, it is possible to open a window that shows its numbered structure, and which $\delta_{\text {SDF }}$ have been matched. Along with it, matched chemical shifts and their corresponding intensity is shown as a graphical representation of a ${ }^{13} \mathrm{C}-\mathrm{NMR}$ spectrum. On the reconstituted spectrum, each carbon type will give a different color and be numbered according to the structure and chemical shift list displayed. This easily shows if the intensities of matched carbons are homogenous, hence hypothetically being signals belonging to the same molecule (Figure S5). It is thus possible for the user to link the information gathered from the structure, the spectrum and the chemical shifts and use his knowledge of NMR spectroscopy to remove or add chemical shifts, artificially modifying the score of the selected molecule. This function can be, for example, used to remove a carbon matched with an abnormal intensity (meaning probably belonging to another molecule), or to add a quaternary carbon that was not matched because it was not picked on the spectrum due to its low intensity, or predicted a bit too far away, etc. If a carbon is added or removed, the spectrum and the highlighted carbons on the structure will be updated accordingly. It is also possible to delete a molecule altogether if necessary. Once the results have been improved by the user, they can be saved as a text and image file (Figure S6). The text file will summarize the parameters used for the research, show the information for each molecule (i.e. name, rank, matched carbons by carbon type). The image file contains the structure of the compounds with the matched carbons highlighted, as well as their name, molecular weight, score and error. 